\title{
Search for an exotic decay of the Higgs boson to a pair of light pseudoscalars in the final state of two muons and two $\tau$ leptons in proton-proton collisions at $\sqrt{s}=13 \mathrm{TeV}$
}

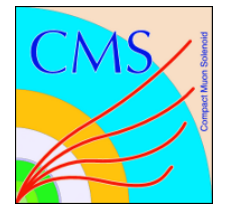

\section{The CMS collaboration}

E-mail: cms-publication-committee-chair@cern.ch

ABSTRACT: A search for exotic Higgs boson decays to light pseudoscalars in the final state of two muons and two $\tau$ leptons is performed using proton-proton collision data recorded by the CMS experiment at the LHC at a center-of-mass energy of $13 \mathrm{TeV}$ in 2016 , corresponding to an integrated luminosity of $35.9 \mathrm{fb}^{-1}$. Masses of the pseudoscalar boson between 15.0 and $62.5 \mathrm{GeV}$ are probed, and no significant excess of data is observed above the prediction of the standard model. Upper limits are set on the branching fraction of the Higgs boson to two light pseudoscalar bosons in different types of two-Higgs-doublet models extended with a complex scalar singlet.

KEYwORDs: Hadron-Hadron scattering (experiments), Higgs physics

ArXIV EPRINT: 1805.04865 


\section{Contents}

1 Introduction 1

2 The CMS detector 2

3 Simulated samples and event reconstruction 3

4 Event selection $\quad 4$

5 Estimation of the background with misidentified $\tau$ leptons 5

$\begin{array}{lll}6 & \text { Signal and background modeling } & 6\end{array}$

$\begin{array}{lll}7 & \text { Systematic uncertainties } & 7\end{array}$

8 Results $\quad 8$

$\begin{array}{lll}9 & \text { Summary } & 10\end{array}$

$\begin{array}{ll}\text { The CMS collaboration } & 17\end{array}$

\section{Introduction}

In 2012 the ATLAS and CMS Collaborations discovered a particle with a mass of $125 \mathrm{GeV}$ [1-3] compatible with the Higgs boson predicted in the standard model (SM) of particle physics [4-9]. Although all the measurements of the couplings and properties of this particle indicate compatibility with the SM within the experimental uncertainties, the existence of exotic decays of the Higgs boson is still allowed. The combination of data collected at center-of-mass energies of 7 and $8 \mathrm{TeV}$ by ATLAS and CMS constrains branching fractions of the Higgs boson to particles beyond the SM to less than $34 \%$ at $95 \%$ confidence level (CL) [10].

Many well-motivated exotic decays of the Higgs boson are proposed in theories beyond the SM [11]. A possible scenario consists of exotic Higgs boson decays to pairs of light pseudoscalars, which subsequently decay to pairs of SM particles. Such a process would be allowed in two-Higgs-doublet models (2HDM) extended with a scalar singlet $(2 \mathrm{HDM}+\mathrm{S})$ [11]. In $2 \mathrm{HDM}+\mathrm{S}, 5$ scalar and 2 pseudoscalar particles are predicted: one of the scalars, h, can be compatible with the discovered Higgs boson, while one of the pseudoscalars, a, can be light enough so that $\mathrm{h} \rightarrow$ aa decays are allowed. The next-to-minimal supersymmetric SM (NMSSM) is a particular case of $2 \mathrm{HDM}+\mathrm{S}[12,13]$.

The ATLAS and CMS Collaborations have set limits on exotic decays of the Higgs boson to a pair of light pseudoscalar bosons, in different final states and in various ranges 
of the pseudoscalar mass, $m_{\mathrm{a}}$ [14-20]. In particular, CMS published a null result in the search in the $2 \mu 2 \tau$ final state for $15.0<m_{\mathrm{a}}<62.5 \mathrm{GeV}$ using data collected at a centerof-mass energy of $8 \mathrm{TeV}$ [14], and ATLAS reported a null result in the same final state at the same energy for $3.7<m_{\mathrm{a}}<50.0 \mathrm{GeV}$ using special reconstruction techniques for Lorentz-boosted $\tau$ lepton pairs [20].

This paper presents a search for an exotic decay of the Higgs boson to a pair of light pseudoscalar bosons in the final state of two muons and two $\tau$ leptons. The analysis is based on data collected in 2016 by the CMS experiment in proton-proton (pp) collisions at a center-of-mass energy of $13 \mathrm{TeV}$, corresponding to an integrated luminosity of $35.9 \mathrm{fb}^{-1}$. Masses of the pseudoscalar boson between 15.0 and $62.5 \mathrm{GeV}$ are probed. Below $15 \mathrm{GeV}$, the pseudoscalar bosons are Lorentz-boosted, causing their decay products to be collimated and to fail the isolation selection criteria used in this analysis. The analysis scans the reconstructed dimuon mass spectrum for a characteristic resonance structure. Four different final states are studied to cover the different possible $\tau$ lepton decay modes: $\mu \mu+\mathrm{e} \mu, \mu \mu+\mathrm{e} \tau_{\mathrm{h}}$, $\mu \mu+\mu \tau_{\mathrm{h}}$, and $\mu \mu+\tau_{\mathrm{h}} \tau_{\mathrm{h}}$, where $\tau_{\mathrm{h}}$ denotes a $\tau$ lepton decaying hadronically. The $\mu \mu+$ ee and $\mu \mu+\mu \mu$ final states are not considered because of their smaller branching fractions and the large background contribution from $\mathrm{Z}$ boson pair production. The event selection and signal extraction used in this analysis have been optimized for the $\mathrm{h} \rightarrow$ aa $\rightarrow 2 \mu 2 \tau$ decay channel, where $\mathrm{h}$ has a mass of $125 \mathrm{GeV}$. Events from the $\mathrm{h} \rightarrow$ aa $\rightarrow 4 \tau$ process can also enter the signal region when at least two of the $\tau$ leptons decay leptonically to muons and neutrinos. These events are treated as a part of the signal even if they do not exhibit a narrow dimuon mass peak. Assuming 2HDM-like scenarios, the ratio of the branching fractions of $\mathrm{a} \rightarrow 2 \mu$ and $\mathrm{a} \rightarrow 2 \tau$ is proportional to the ratio of the squared masses of the muon and the $\tau$ lepton:

$$
\frac{\mathcal{B}(\mathrm{a} \rightarrow 2 \mu)}{\mathcal{B}(\mathrm{a} \rightarrow 2 \tau)}=\frac{m_{\mu}^{2} \sqrt{1-\left(2 m_{\mu} / m_{\mathrm{a}}\right)^{2}}}{m_{\tau}^{2} \sqrt{1-\left(2 m_{\tau} / m_{\mathrm{a}}\right)^{2}}} \simeq \frac{m_{\mu}^{2}}{m_{\tau}^{2}} .
$$

Events are selected only if the invariant mass of the four objects in the final state is below $100-130 \mathrm{GeV}$ (depending on the final state) to enforce the compatibility with a Higgs boson decay. This criterion strongly suppresses both the background from events with genuine leptons, which arise mostly from the $\mathrm{Z}$ boson pair production, and the backgrounds with jets misidentified as $\tau$ leptons, leaving only a few expected background events in the signal region. The background from $\mathrm{Z}$ boson pair production is estimated from simulation, whereas the background with jets misidentified as $\tau$ leptons is estimated from data, as detailed in section 5. The presence of a signal is probed using the reconstructed dimuon mass as an observable. Given the narrow width of the signal and the small number of expected background events, signal and background distributions are parameterized to perform an unbinned maximum-likelihood fit.

\section{The CMS detector}

The central feature of the CMS apparatus is a superconducting solenoid of $6 \mathrm{~m}$ internal diameter, providing a magnetic field of $3.8 \mathrm{~T}$. Within the solenoid volume, there are a silicon 
pixel and strip tracker, a lead tungstate crystal electromagnetic calorimeter (ECAL), and a brass and scintillator hadron calorimeter, each composed of a barrel and two endcap sections. Forward calorimeters extend the pseudorapidity coverage provided by the barrel and endcap detectors. Muons are detected in gas-ionization chambers embedded in the steel flux-return yoke outside the solenoid. Events of interest are selected using a twotiered trigger system [21]. A more detailed description of the CMS detector, together with a definition of the coordinate system used and the relevant kinematic variables, can be found in ref. [22].

\section{Simulated samples and event reconstruction}

Signal processes, for both $\mathrm{h} \rightarrow$ aa $\rightarrow 2 \mu 2 \tau$ and $\mathrm{h} \rightarrow$ aa $\rightarrow 4 \tau$, are generated using the MADGRAPH5_aMC@NLO 2.2.2 generator [23] with its implementation of the 2HDM and the NMSSM, in gluon fusion and vector boson fusion production. They are simulated at leading order (LO) in perturbative quantum chromodynamics (QCD) with the MLM jet matching and merging scheme [24]. The generator is interfaced with PYTHIA 8.212 [25] to model the parton showering and fragmentation as well as the decay of the $\tau$ leptons. The CUETP8M1 tune [26] is chosen for the PYTHIA parameters controlling the description of the underlying event. The ZZ background from quark-antiquark annihilation is generated at next-to-LO (NLO) in perturbative QCD with POWHEG v2.0 [27-29], while the gg $\rightarrow$ ZZ process is generated at LO with MCFM 7.0 [30]. The set of parton distribution functions is NLO NNPDF3.0 for NLO samples, and LO NNPDF3.0 for LO samples [31]. The fully differential cross section for the $\mathrm{q} \overline{\mathrm{q}} \rightarrow \mathrm{ZZ}$ process has been computed at next-to-NLO (NNLO) [32], and the NNLO/NLO $K$-factor is applied to the POWHEG sample as a function of the invariant mass of the $\mathrm{Z}$ boson pair. Rare processes, such as triboson, $\mathrm{t} \overline{\mathrm{tZ}}$, or SM Higgs boson production, have a negligible contribution to the signal region because they typically have a larger invariant mass of the four leptons in the final state.

Simulated samples include additional pp interactions per bunch crossing (pileup), and are reweighted so as to match the pileup distribution observed in data. Generated events are processed through a simulation of the CMS detector based on GEANT4 [33].

The reconstruction of events relies on the particle-flow (PF) algorithm [34], which combines the information from the CMS subdetectors to identify and reconstruct the particles emerging from pp collisions: charged and neutral hadrons, photons, muons, and electrons. Combinations of these PF objects are used to reconstruct higher-level objects such as jets or $\tau_{\mathrm{h}}$ candidates. The reconstructed vertex with the largest value of summed physics-object $p_{\mathrm{T}}^{2}$ is taken to be the primary pp interaction vertex, where $p_{\mathrm{T}}$ denotes the transverse momentum. The physics objects are the jets, clustered using a jet-finding algorithm $[35,36]$ with the tracks assigned to the vertex as inputs, and the associated missing transverse momentum, taken as the negative vector sum of the $p_{\mathrm{T}}$ of those jets.

Electrons are reconstructed by matching ECAL clusters to tracks in the tracker. They are then identified with a multivariate discriminant that makes use of variables related to energy deposits in the ECAL, to the quality of the track, and to the compatibility between the ECAL clusters and the track that have been matched together [37]. Muons 
are reconstructed by building tracks from hits in the tracker and in the muon system, and are identified using variables related to the number of measurements in the tracker and the muon systems and to the quality of the track reconstruction [38]. They are required to have a relative isolation less than 0.2 , with the relative isolation variable defined as follows:

$$
I^{\mu} \equiv \frac{\sum_{\text {charged }} p_{\mathrm{T}}+\max \left(0, \sum_{\text {neutral }} p_{\mathrm{T}}-\frac{1}{2} \sum_{\text {charged, PU }} p_{\mathrm{T}}\right)}{p_{\mathrm{T}}^{\mu}} .
$$

In this equation, $\sum_{\text {charged }} p_{\mathrm{T}}$ is the scalar $p_{\mathrm{T}}$ sum of the charged particles associated with the primary vertex in a cone of size $\Delta R=\sqrt{(\Delta \eta)^{2}+(\Delta \phi)^{2}}=0.4$ around the muon direction. The sum $\sum_{\text {neutral }} p_{\mathrm{T}}$ is a similar quantity for neutral particles. The $p_{\mathrm{T}}$ of neutral particles originating from pileup vertices is considered on the basis of simulation to be half of that of charged particles associated with pileup vertices, denoted by $\sum_{\text {charged, } \mathrm{PU}} p_{\mathrm{T}}$. The term $p_{\mathrm{T}}^{\mu}$ denotes the muon $p_{\mathrm{T}}$. The azimuthal angle, $\phi$, is expressed in radians.

Jets are reconstructed from $\mathrm{PF}$ objects with the anti- $k_{\mathrm{T}}$ clustering algorithm implemented in the FASTJET library [36, 39], using a distance parameter of 0.4. Jets that originate from $\mathrm{b}$ quarks, called $\mathrm{b}$ jets, are identified with the combined secondary vertex (CSVv2) algorithm [40]. The algorithm builds a discriminant from variables related to potential secondary vertices associated to the jet, and from track-based lifetime information. The working point chosen in this search provides an efficiency for b quark jets of approximately $70 \%$, and a misidentification rate for light-flavor jets of approximately $1 \%$. Events with reconstructed $\mathrm{b}$ jets with $p_{\mathrm{T}}>20 \mathrm{GeV}$ are vetoed in this analysis to reject $\mathrm{t} \overline{\mathrm{t}}$ events and other backgrounds with $\mathrm{b}$ quark jets.

Hadronically decaying $\tau$ leptons are reconstructed with the hadrons-plus-strips algorithm $[41,42]$. This algorithm starts from anti- $k_{\mathrm{T}}$ jets and reconstructs $\tau_{\mathrm{h}}$ candidates from tracks and energy deposits in strips of the ECAL, in the 1-prong, 1-prong $+\pi^{0}$, 2-prong, and 3-prong decay modes. The 2-prong decay mode allows $\tau_{\mathrm{h}}$ candidates to be reconstructed even if one track has not been reconstructed. Given the large rate for jets to be misidentified in this decay mode and the limited increase in efficiency for genuine $\tau_{\mathrm{h}}$ candidates, the 2-prong decay mode is not used to reconstruct $\tau_{\mathrm{h}}$ candidates in the signal region of this analysis, but is used in some control regions to study events with jets misidentified as $\tau_{\mathrm{h}}$ candidates. Hadronically decaying $\tau$ leptons are further required to be identified using a multivariate discriminator that combines isolation and lifetime variables. The working point of the discriminator has a $\tau_{\mathrm{h}}$ identification efficiency of approximately $57 \%$ for a misidentification rate of light-flavor jets of approximately $0.35 \%$. Discriminators to reject muons and electrons misidentified as $\tau_{\mathrm{h}}$ candidates are further applied.

\section{Event selection}

Online, events are required to pass a double-muon trigger with $p_{\mathrm{T}}$ thresholds of 17 and $8 \mathrm{GeV}$ for the leading and subleading muons, respectively, or a single-muon trigger with a $p_{\mathrm{T}}$ threshold of $24 \mathrm{GeV}$. In the $\mu \mu+\mathrm{e} \mu$ and $\mu \mu+\mu \tau_{\mathrm{h}}$ final states, events are also selected if they pass a triple-muon trigger with $p_{\mathrm{T}}$ thresholds of 12,10 , and $5 \mathrm{GeV}$. Offline, the leading 
muon must have $p_{\mathrm{T}}>18 \mathrm{GeV}$ (or $25 \mathrm{GeV}$ if only the single-muon trigger is satisfied), and the subleading one $p_{\mathrm{T}}>9 \mathrm{GeV}$ (or $11 \mathrm{GeV}$ if only the triple-muon trigger is satisfied). Selecting muons offline with $p_{\mathrm{T}}$ thresholds $1 \mathrm{GeV}$ above the online thresholds ensures fully efficient triggers in this analysis. If there are additional muons, each is required to have $p_{\mathrm{T}}>5 \mathrm{GeV}$ (or $6 \mathrm{GeV}$ if only the triple-muon trigger conditions have been met). All muons must satisfy $|\eta|<2.4$. Electrons from $\tau$ lepton decays are required to have $p_{\mathrm{T}}>7 \mathrm{GeV}$ and $|\eta|<2.5$, and $\tau_{\mathrm{h}}$ candidates are required to satisfy $p_{\mathrm{T}}>18.5 \mathrm{GeV}$ and $|\eta|<2.3$. Each event is required to have an opposite-sign (OS) pair of isolated muons and an OS pair of isolated $\tau$ candidates (e, $\mu$, or $\tau_{\mathrm{h}}$ ).

In final states with three muons, the highest $p_{\mathrm{T}}$ muon is considered as originating promptly from the decay of the pseudoscalar bosons. It is paired with the next-highest $p_{\mathrm{T}}$ OS muon. The third muon is considered as a decay product of a $\tau$ lepton. The probability for success of this algorithm for the expected signal varies between 72 and $94 \%$, and increases with the pseudoscalar boson mass.

The overlap between the events selected in the four different final states is removed: events that have more isolated muons or electrons than those needed to build the fourlepton final state under study are discarded from the analysis in that final state. Selected leptons are required to be separated from each other by $\Delta R>0.3$, or $>0.4$ if there is a $\tau_{\mathrm{h}}$ candidate, since it is built from a jet with a distance parameter of $\Delta R=0.4$.

More than $80 \%$ of the background is rejected by keeping only events for which the visible invariant mass of the four leptons is below $110 \mathrm{GeV}$ in the $\mu \mu+\mathrm{e} \mu$ final state, $120 \mathrm{GeV}$ in the $\mu \mu+\mathrm{e} \tau_{\mathrm{h}}$ and $\mu \mu+\mu \tau_{\mathrm{h}}$ final states, and $130 \mathrm{GeV}$ in the $\mu \mu+\tau_{\mathrm{h}} \tau_{\mathrm{h}}$ final state. The threshold depends on the final state because of the different number of neutrinos from $\tau$ lepton decays. Because of the neutrinos, the visible invariant mass is expected to peak below $125 \mathrm{GeV}$ for the signal, and this selection criterion has a signal efficiency close to $100 \%$. Additionally, the visible mass of the $\tau \tau$ pair is required to be smaller than the dimuon mass. Events that have a reconstructed dimuon mass lower than $14 \mathrm{GeV}$ or higher than $64 \mathrm{GeV}$ are rejected from the signal region.

The selection described above is optimized for the $\mathrm{h} \rightarrow$ aa $\rightarrow 2 \mu 2 \tau$ signal process, which benefits from an excellent dimuon mass resolution of the CMS detector. Assuming a $2 \mathrm{HDM}+\mathrm{S}$ model, the yield of the $\mathrm{h} \rightarrow$ aa $\rightarrow 4 \tau$ signal after the selection is between 13 and $52 \%$ of all $\mathrm{h} \rightarrow$ aa signal events, depending on the final state. The largest fraction is obtained in the $\mu \mu+\mathrm{e} \mu$ final state, where the lepton $p_{\mathrm{T}}$ thresholds are the lowest, while the lowest fraction appears in the $\mu \mu+\tau_{\mathrm{h}} \tau_{\mathrm{h}}$ final state, which has the highest lepton $p_{\mathrm{T}}$ thresholds.

\section{Estimation of the background with misidentified $\tau$ leptons}

The background composed of events where at least one jet is misidentified as one of the final-state leptons is estimated from data. Such events include mostly Z+jets and WZ+jets events, but there are also minor contributions from $\mathrm{ZZ} \rightarrow 2 \ell 2 \mathrm{q}$ events, $\mathrm{t} \overline{\mathrm{t}}$ production, or from the background from SM events comprised uniquely of jets produced through the 
strong interaction, referred to as QCD multijet events. The yield and the distributions of these backgrounds are estimated from data via a two-step procedure:

1. The shape is obtained from data in a signal and ZZ background free control region with the $\tau$ candidates of same sign (SS). To increase the statistical precision of the templates and enrich the region in events with jets misidentified as leptons, the isolation criteria on the $\tau$ candidates are relaxed and $\tau_{\mathrm{h}}$ candidates are allowed to be also reconstructed as 2-prong decays. Including the 2-prong decays increases the data yield in the control region by about $50 \%$.

2. The yield is estimated from data events that have one or two nonisolated $\tau$ candidates. These events are reweighted with factors that describe the probability for jets to pass the isolation criteria used to select the $\tau$ candidates. The misidentification probabilities for jets are measured in $\mathrm{Z} \rightarrow \mu \mu+$ jets events, selected with the same selection criteria as in the signal region except that neither isolation, nor identification criteria are applied to the $\tau$ candidates, which are further required to have SS. Additionally the dimuon pair is required to have an invariant mass between 70 and $110 \mathrm{GeV}$. The probabilities are measured separately in the barrel and in the endcaps as a function of the $p_{\mathrm{T}}$ of the jet that is closest to the lepton, and are parameterized with Landau functions.

The estimation method for the background with jets misidentified as leptons is validated in three control regions: one containing events that pass the full signal selection except that the four-lepton mass criterion is inverted; another where $\tau_{\mathrm{h}}$ candidates are reconstructed as 2-prong decays only; and a third one with two SS $\tau$ candidates. The background predictions and data are statistically compatible, with deviations not exceeding $20-40 \%$ depending on the final state. The background estimation method has also been validated in simulation for $\mathrm{WZ}+$ jets and $\mathrm{Z}+$ jets events.

\section{Signal and background modeling}

The results are extracted by fitting the reconstructed dimuon mass distributions. The dimuon mass distributions of the simulated $\mathrm{h} \rightarrow$ aa $\rightarrow 2 \mu 2 \tau$ signal events passing all selection criteria are parameterized with Voigt functions, which are convolutions of the Gaussian and Lorentzian profiles with a common mean. The parameterizations for different $m_{\mathrm{a}}$ values in the $\mu \mu+\mu \tau_{\mathrm{h}}$ final state are shown in figure 1 (left). The dimuon mass resolution is better than $2 \%$ for all masses and final states considered in the analysis. The parameters of the Voigt functions are fit for each simulated mass and for each final state. The parameters are interpolated for signal masses not covered by simulation.

For the $\mathrm{h} \rightarrow$ aa $\rightarrow 4 \tau$ signal, the two reconstructed muons that have been chosen to form the dimuon mass distribution can come from either pseudoscalar boson. When the two muons come from the same boson, their visible mass distribution is a wide peak below $m_{\mathrm{a}}$ because they originate from $\tau$ lepton decays. When the two muons come from different bosons, they do not form a resonance and their mass distribution is rather flat, with a 

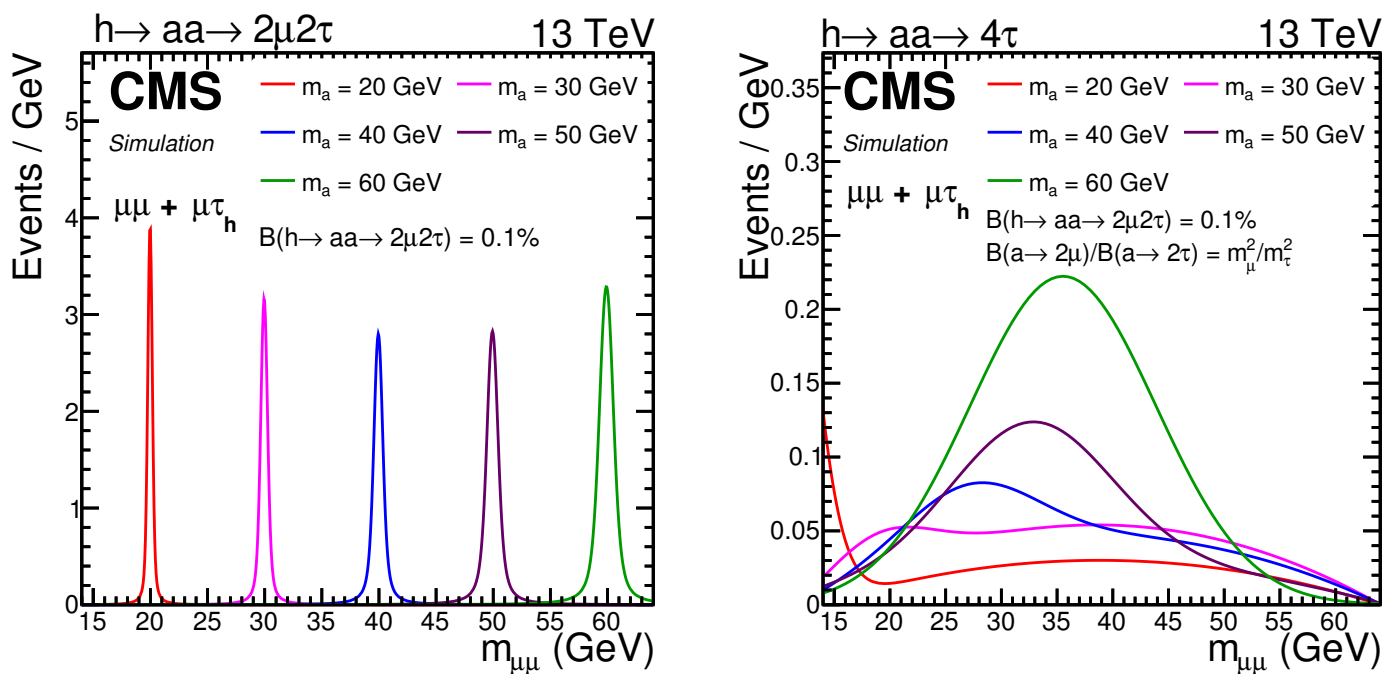

Figure 1. Parameterized dimuon invariant mass distributions of the $\mathrm{h} \rightarrow$ aa $\rightarrow 2 \mu 2 \tau$ (left) and $\mathrm{h} \rightarrow$ aa $\rightarrow 4 \tau$ (right) signal processes simulated at different $m_{\mathrm{a}}$ values in the $\mu \mu+\mu \tau_{\mathrm{h}}$ final state. The normalization corresponds to the number of expected signal events after the selection for an integrated luminosity of $35.9 \mathrm{fb}^{-1}$, assuming the production cross section of the Higgs boson predicted in the $\mathrm{SM}$, and $\mathcal{B}(\mathrm{h} \rightarrow$ aa $\rightarrow 2 \mu 2 \tau)=2 \mathcal{B}(\mathrm{h} \rightarrow \mathrm{aa}) \mathcal{B}(\mathrm{a} \rightarrow \mu \mu) \mathcal{B}(\mathrm{a} \rightarrow \tau \tau)=0.1 \%$. The yield of the $\mathrm{h} \rightarrow$ aa $\rightarrow 4 \tau$ contribution is further rescaled according to the relation in eq. (1.1).

shape sculpted by kinematic selections. The dimuon mass distribution of the $\mathrm{h} \rightarrow$ aa $\rightarrow 4 \tau$ signal is parameterized with the sum of a Gaussian function for the resonant contribution and of a polynomial for the nonresonant contribution. The parameterizations for different $m_{\mathrm{a}}$ values in the $\mu \mu+\mu \tau_{\mathrm{h}}$ final state are shown in figure 1 (right).

The dimuon mass distributions of the $\mathrm{Z}$ pair background and the background with misidentified $\tau$ leptons are parameterized with Bernstein polynomials. The number of degrees of the polynomial required to describe the background in each channel is determined with a Fisher $F$-test [43], which selects the minimal number that allows for a good fit quality. The parameterizations of the backgrounds in the $\mu \mu+\mu \tau_{\mathrm{h}}$ final state are shown in figure 2. The choice of the fit function and of its degree has only a limited impact on the final results because of the low expected background yields.

\section{Systematic uncertainties}

Yield uncertainties for the processes estimated from simulation include the uncertainty in the integrated luminosity (2.5\%) [44], in the trigger efficiency $(2 \%)$, and in the vetoing of b-tagged jets (0.5\%). Additionally, the identification, isolation, and reconstruction uncertainties amount to $2 \%$ per muon, $2 \%$ per electron, and $5 \%$ per $\tau_{\mathrm{h}}$ candidate. The uncertainty in the $\tau_{\mathrm{h}}$ energy scale leads to yield uncertainties between 1 and $2 \%$. The uncertainty in the yield of the $\mathrm{ZZ}$ background is $12 \%$ : it accounts for the uncertainties in the renormalization and factorization scales, as well as for the uncertainty related to the absence of higher-order electroweak corrections in simulation. The statistical uncertainty related to the limited size of the $\mathrm{ZZ}$ simulated sample reaches up to $13 \%$ in the $\mu \mu+\tau_{\mathrm{h}} \tau_{\mathrm{h}}$ 

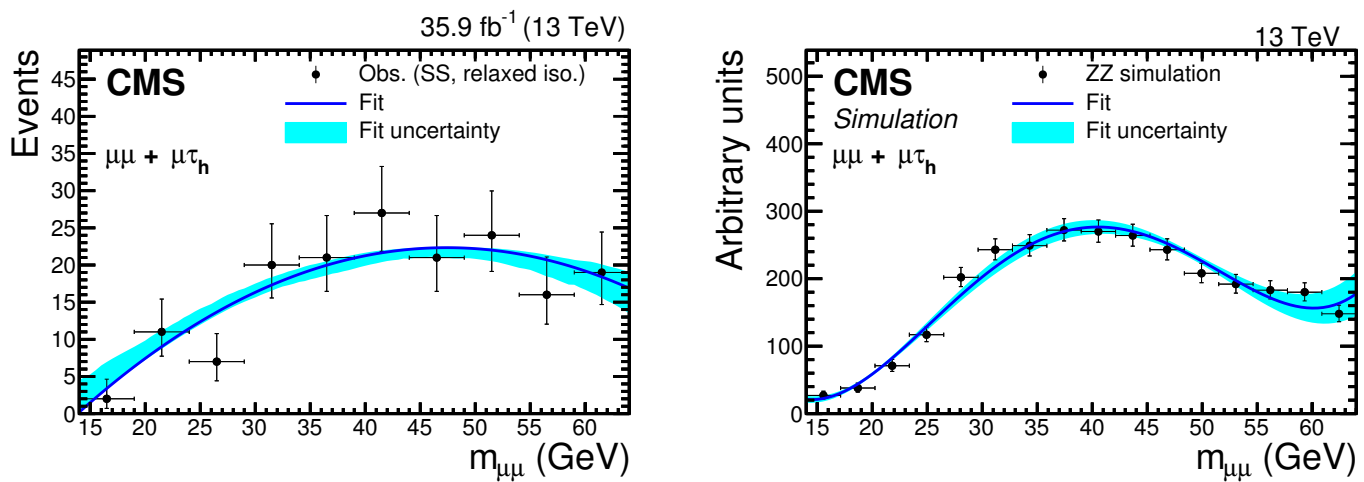

Figure 2. Parameterization of the shape of the background with misidentified $\tau$ leptons (left) and $\mathrm{Z}$ pair production background (right) in the $\mu \mu+\mu \tau_{\mathrm{h}}$ final state. The points for the ZZ background represent events selected in simulation, whereas they correspond to observed data events in the SS region with relaxed isolation for the background with misidentified $\tau$ leptons.

final state, but is well below $3 \%$ in the other final states. The uncertainty in the normalization of the signal shapes arising from the parameterization of the normalization as a function of the mass is $5 \%$ per final state. The shape uncertainties related to the parameterization of the signal consist of a $0.1 \%$ uncertainty in the mean of the Voigt profile and an anticorrelated $30 \%$ uncertainty in the two width parameters.

The yield uncertainty in the background with jets misidentified as $\tau$ leptons accounts for two different components: the level of agreement between data and background prediction in the control regions, and the statistical uncertainty in the yield predicted in the signal region. As discussed in section 5, the first component varies between 20 and 40\%, depending on the final state, whereas the second one ranges between 11 and $23 \%$. The uncertainties in the parameters of the polynomials used to parameterize the distributions of the background with jets misidentified as $\tau$ leptons are included as nuisance parameters in the fit. These parameter uncertainties are obtained from the fits to the data control regions with same sign $\tau$ candidates passing relaxed isolation and reconstruction conditions. The uncertainty related to the choice of the fit function for the backgrounds is negligible with respect to the size of the statistical uncertainty. This has been verified by comparing the expected upper limits on the signal when other functional forms are chosen to parameterize the backgrounds.

\section{Results}

To test for the existence of a resonance, an unbinned maximum-likelihood fit to the dimuon invariant mass distribution is performed. In the fit, the systematic uncertainties are nuisance parameters varied according to a log-normal probability density function for the yield uncertainties and a Gaussian probability density function for the shape uncertainties. The dimuon mass distributions for the four final states are shown in figure 3. The expected background and signal yields in the signal region are given in table 1 for the four final states. 

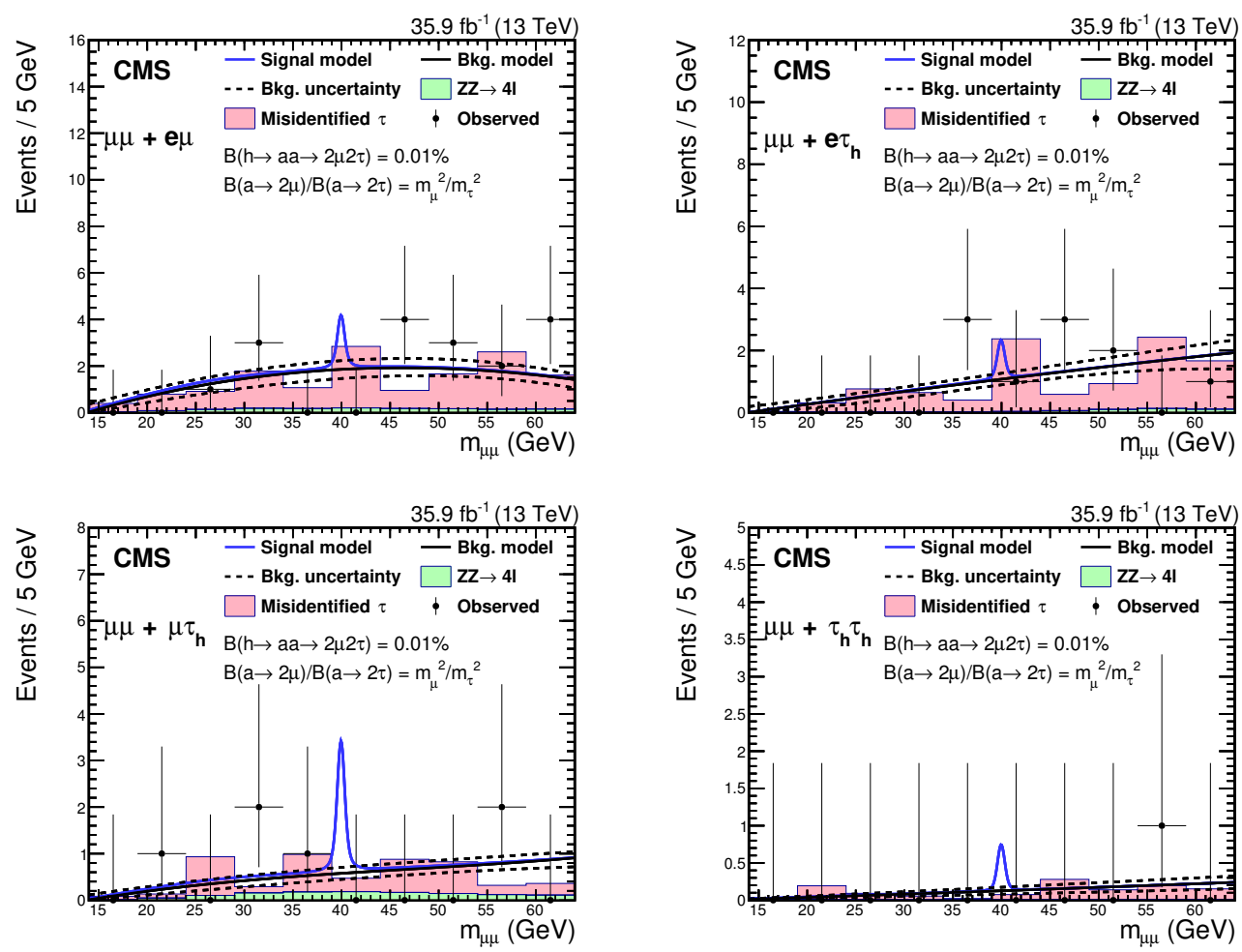

Figure 3. Dimuon mass distributions in the $\mu \mu+\mathrm{e} \mu$ (upper left), $\mu \mu+\mathrm{e} \tau_{\mathrm{h}}$ (upper right), $\mu \mu+$ $\mu \tau_{\mathrm{h}}$ (lower left), and $\mu \mu+\tau_{\mathrm{h}} \tau_{\mathrm{h}}$ (lower right) final states. The total background estimate and its uncertainty are given by the black lines. The histograms for the two background components are shown for illustrative purposes only as the background models are extracted from unbinned fits. The signal model is drawn in blue above the background model: it includes both $\mathrm{h} \rightarrow$ aa $\rightarrow 2 \mu 2 \tau$ and $\mathrm{h} \rightarrow$ aa $\rightarrow 4 \tau$, and is normalized using $\mathcal{B}(\mathrm{h} \rightarrow$ aa $\rightarrow 2 \mu 2 \tau)=0.01 \%$, assuming the relation in eq. (1.1) to determine the relative proportion of these processes. The production cross section of the Higgs boson predicted in the SM is assumed.

\begin{tabular}{|lcccc|}
\hline & $\mu \mu+\mathrm{e} \mu$ & $\mu \mu+\mathrm{e} \tau_{\mathrm{h}}$ & $\mu \mu+\mu \tau_{\mathrm{h}}$ & $\mu \mu+\tau_{\mathrm{h}} \tau_{\mathrm{h}}$ \\
\hline $\mathrm{ZZ} \rightarrow 4 \ell$ & $1.5 \pm 0.2$ & $0.5 \pm 0.1$ & $1.2 \pm 0.2$ & $0.03 \pm 0.01$ \\
Misidentified $\tau$ & $13.2 \pm 5.5$ & $9.7 \pm 2.5$ & $4.0 \pm 1.2$ & $1.2 \pm 0.5$ \\
$\mathrm{~h} \rightarrow \mathrm{aa} \rightarrow 2 \mu 2 \tau, m_{\mathrm{a}}=20 \mathrm{GeV}$ & 0.39 & 0.25 & 0.47 & 0.10 \\
$\mathrm{~h} \rightarrow \mathrm{aa} \rightarrow 4 \tau, m_{\mathrm{a}}=20 \mathrm{GeV}$ & 0.37 & 0.04 & 0.24 & 0.01 \\
$\mathrm{~h} \rightarrow$ aa $\rightarrow 2 \mu 2 \tau, m_{\mathrm{a}}=40 \mathrm{GeV}$ & 0.57 & 0.28 & 0.68 & 0.14 \\
$\mathrm{~h} \rightarrow$ aa $\rightarrow 4 \tau, m_{\mathrm{a}}=40 \mathrm{GeV}$ & 0.68 & 0.09 & 0.48 & 0.02 \\
$\mathrm{~h} \rightarrow$ aa $\rightarrow 2 \mu 2 \tau, m_{\mathrm{a}}=60 \mathrm{GeV}$ & 0.94 & 0.85 & 1.18 & 0.52 \\
$\mathrm{~h} \rightarrow$ aa $\rightarrow 4 \tau, m_{\mathrm{a}}=60 \mathrm{GeV}$ & 1.27 & 0.20 & 0.93 & 0.05 \\
Observed & 17 & 10 & 6 & 1 \\
\hline
\end{tabular}

Table 1. Yields of the signal and background processes in the four final states, as well as the number of observed events in each final state, in the dimuon mass range between 14 and $64 \mathrm{GeV}$. The signal yields are given for $\mathcal{B}(\mathrm{h} \rightarrow$ aa $\rightarrow 2 \mu 2 \tau)=0.01 \%$. The $\mathrm{h} \rightarrow$ aa $\rightarrow 4 \tau$ signal is scaled assuming the couplings of the pseudoscalar boson proportional to the squared lepton mass, as in eq. (1.1). The production cross section of the Higgs boson predicted in the SM is assumed. The uncertainties combine the statistical and systematic sources. 
No significant excess of data is observed above the expected SM background. Upper limits at $95 \%$ CL are set on $\left(\sigma_{\mathrm{h}} / \sigma_{\mathrm{SM}}\right) \mathcal{B}(\mathrm{h} \rightarrow$ aa $\rightarrow 2 \mu 2 \tau)=2\left(\sigma_{\mathrm{h}} / \sigma_{\mathrm{SM}}\right) \mathcal{B}(\mathrm{h} \rightarrow$ aa $) \mathcal{B}(\mathrm{a} \rightarrow$ $\mu \mu) \mathcal{B}(\mathrm{a} \rightarrow \tau \tau)$ using the modified frequentist construction $\mathrm{CL}_{\mathrm{s}}[45-48]$ for pseudoscalar masses between 15.0 and $62.5 \mathrm{GeV}$. In this expression, $\sigma_{\mathrm{h}} / \sigma_{\mathrm{SM}}$ is the Higgs boson cross section for the gluon fusion and vector boson fusion production modes, divided by its SM prediction. The limits are shown in figure 4 for the individual final states and for their combination. The combined upper limits on the branching fraction $\mathcal{B}(\mathrm{h} \rightarrow$ aa $\rightarrow 2 \mu 2 \tau)$ are as low as $1.2 \times 10^{-4}$ for a mass of $60 \mathrm{GeV}$ assuming the SM production cross section for the Higgs boson. The expected limits are the tightest for the $\mu \mu+\mu \tau_{\mathrm{h}}$ final state because the lepton $p_{\mathrm{T}}$ thresholds are lower than in the $\mu \mu+\mathrm{e} \tau_{\mathrm{h}}$ and $\mu \mu+\tau_{\mathrm{h}} \tau_{\mathrm{h}}$ final states, and because the branching fraction is larger than in the $\mu \mu+\mathrm{e} \mu$ final state. The $\mathrm{h} \rightarrow \mathrm{aa} \rightarrow 4 \tau$ signal is assumed to scale according to eq. (1.1) with respect to the $\mathrm{h} \rightarrow$ aa $\rightarrow 2 \mu 2 \tau$ signal. Alternatively, considering a null contribution from $\mathrm{h} \rightarrow$ aa $\rightarrow 4 \tau$, there is still no significant excess of data over the expected SM background and the expected limits become less stringent by approximately $10 \%$.

The results can be interpreted as upper limits on $\left(\sigma_{\mathrm{h}} / \sigma_{\mathrm{SM}}\right) \mathcal{B}(\mathrm{h} \rightarrow$ aa) in the different $2 \mathrm{HDM}+\mathrm{S}$ models. Types I-IV $2 \mathrm{HDM}+\mathrm{S}$ forbid flavor changing neutral currents at tree level. In type I $2 \mathrm{HDM}+\mathrm{S}$, all SM particles couple to the first doublet and the branching fractions of the light pseudoscalar to SM particles are independent of $\tan \beta$, defined as the ratio of the vacuum expectation value of the second doublet to that of the first doublet. In type II $2 \mathrm{HDM}+\mathrm{S}$, including the NMSSM, up-type quarks couple to the first doublet, and leptons and down-type quarks couple to the second doublet. This leads to pseudoscalar decays to leptons and down-type fermions enhanced for $\tan \beta>1$. In these two types, the analysis is sensitive to a cross section larger than approximately three times the SM production cross section of the Higgs boson for $\mathcal{B}(\mathrm{h} \rightarrow$ aa $\rightarrow 2 \mu 2 \tau)=100 \%$. In type III $2 \mathrm{HDM}+\mathrm{S}$, quarks couple to the first doublet and leptons to the second one, making it the most favorable type of $2 \mathrm{HDM}+\mathrm{S}$ for $\mathrm{h} \rightarrow$ aa $\rightarrow 2 \mu 2 \tau$ decays at large $\tan \beta$. In type IV $2 \mathrm{HDM}+\mathrm{S}$, leptons and up-type quarks couple to the first doublet while down-type quarks couple to the second doublet. With $m_{\mathrm{a}}, \tan \beta$, and the type of $2 \mathrm{HDM}+\mathrm{S}$ specified, the branching fractions of the pseudoscalars to SM particles can be predicted following the prescriptions in refs. [11, 49]. The results expressed as limits on $\left(\sigma_{\mathrm{h}} / \sigma_{\mathrm{SM}}\right) \mathcal{B}(\mathrm{h} \rightarrow$ aa $)$ are shown in figure 5 for the last two types of $2 \mathrm{HDM}+\mathrm{S}$. The most stringent limits are obtained in $2 \mathrm{HDM}+\mathrm{S}$ type III at large $\tan \beta$, where the couplings to leptons are enhanced, and where limits of approximately $3 \%$ are set for $\tan \beta \gtrsim 3$. This analysis improves previous results [14] in the $2 \mu 2 \tau$ final state by a factor two or more for $15.0<m_{\mathrm{a}}<62.5 \mathrm{GeV}$ in all four types of $2 \mathrm{HDM}+\mathrm{S}$.

\section{Summary}

A search for an exotic decay of the Higgs boson to a pair of light pseudoscalars in the final state of two muons and two $\tau$ leptons has been performed using data collected by the CMS experiment in 2016 at a center-of-mass energy of $13 \mathrm{TeV}$, and corresponding to an integrated luminosity of $35.9 \mathrm{fb}^{-1}$. The results are extracted from an unbinned fit of the dimuon mass spectrum. Limits are set at $95 \%$ confidence level on the branching fraction 

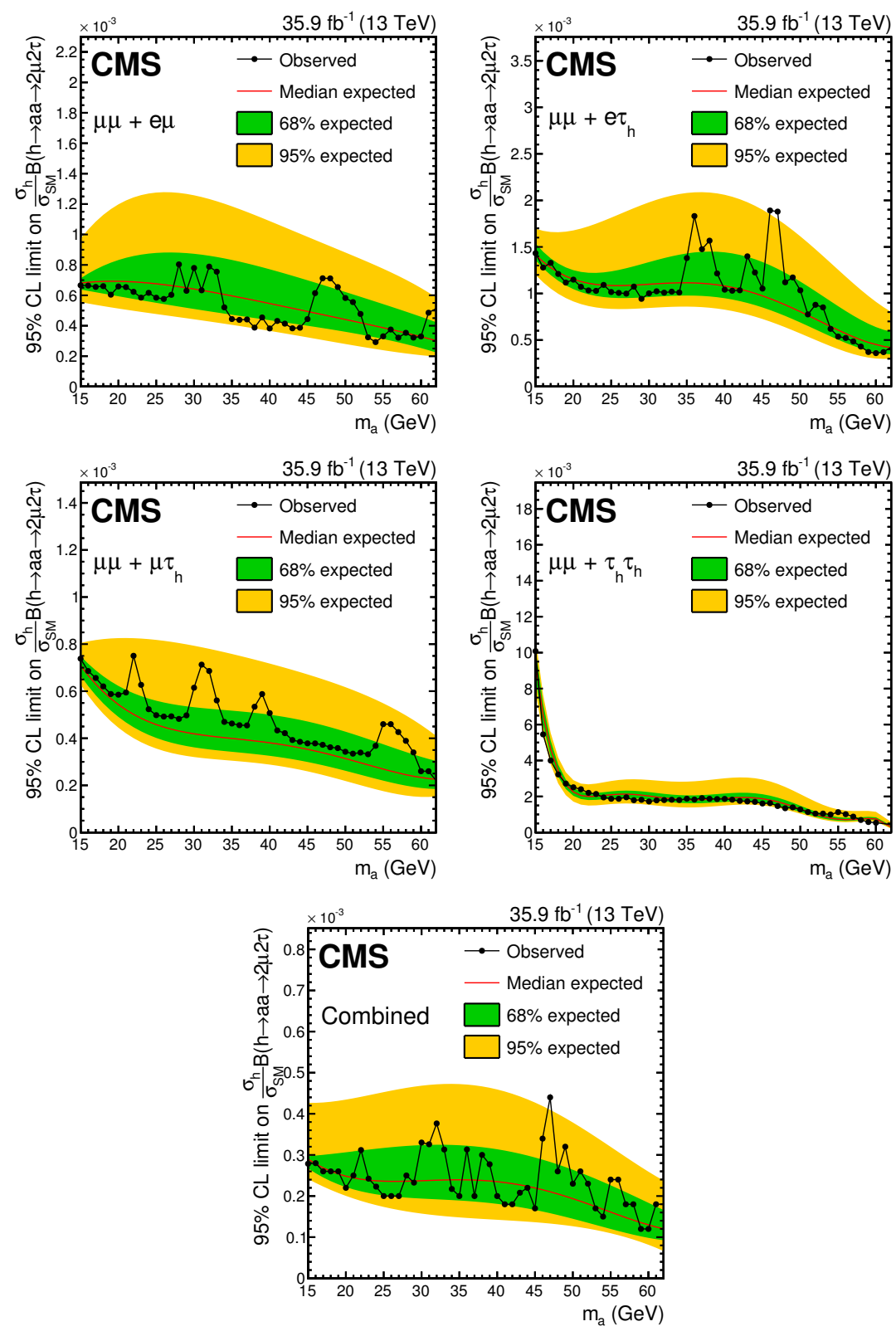

Figure 4. Upper limits at $95 \% \mathrm{CL}$ on $\left(\sigma_{\mathrm{h}} / \sigma_{\mathrm{SM}}\right) \mathcal{B}(\mathrm{h} \rightarrow \mathrm{aa} \rightarrow 2 \mu 2 \tau)$, in the $\mu \mu+\mathrm{e} \mu$ (upper left), $\mu \mu+\mathrm{e} \tau_{\mathrm{h}}$ (upper right), $\mu \mu+\mu \tau_{\mathrm{h}}$ (middle left), $\mu \mu+\tau_{\mathrm{h}} \tau_{\mathrm{h}}$ (middle right) final states, and for the combination of these final states (lower). The $\mathrm{h} \rightarrow$ aa $\rightarrow 4 \tau$ process is considered as a part of the signal, and is scaled with respect to the $\mathrm{h} \rightarrow$ aa $\rightarrow 2 \mu 2 \tau$ signal using eq. (1.1).

$\mathcal{B}(\mathrm{h} \rightarrow$ aa $\rightarrow 2 \mu 2 \tau)$ for the masses of the light pseudoscalar between 15.0 and $62.5 \mathrm{GeV}$, and are as low as $1.2 \times 10^{-4}$ for a mass of $60 \mathrm{GeV}$ assuming the SM production cross section for the Higgs boson. These are the most stringent limits obtained in the final state of two muons and two $\tau$ leptons for the masses above $15 \mathrm{GeV}$, improving previous limits [14, 20] by more than a factor two. They provide the tightest constraints in this mass range on exotic Higgs boson decays in scenarios where the decays of pseudoscalar bosons to leptons are enhanced. 

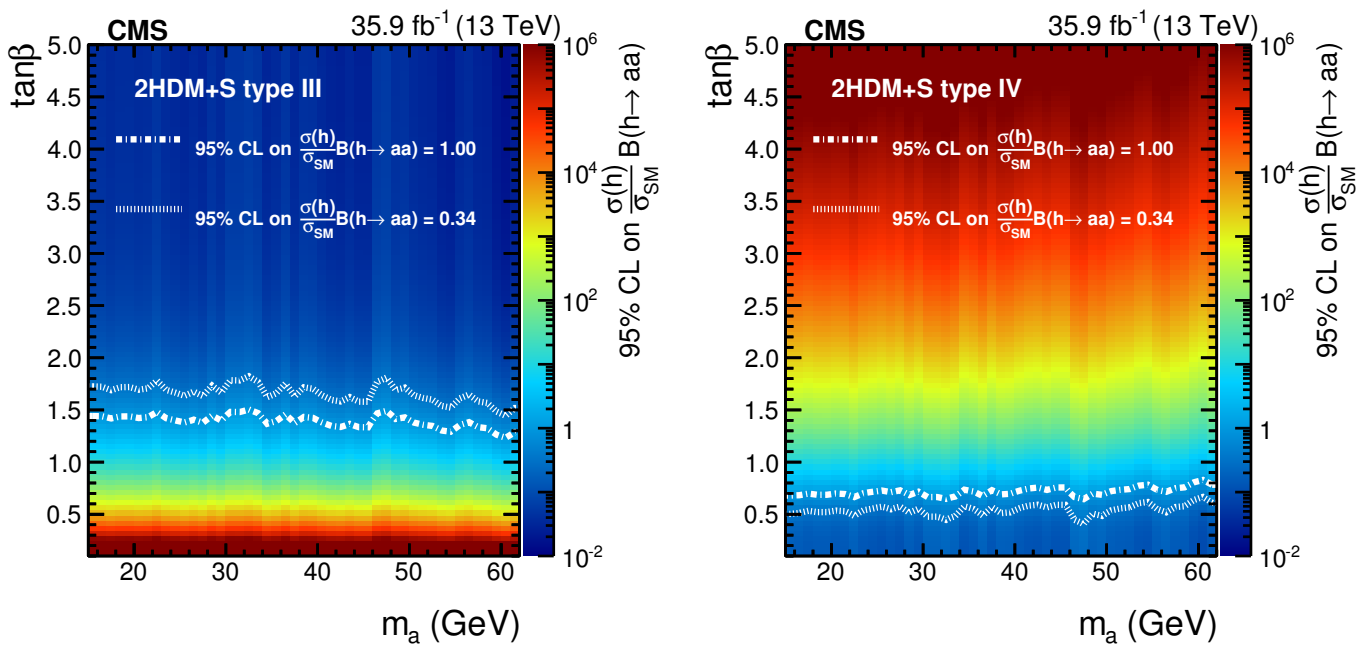

Figure 5. Observed limits on $\left(\sigma_{\mathrm{h}} / \sigma_{\mathrm{SM}}\right) \mathcal{B}(\mathrm{h} \rightarrow \mathrm{aa})$ in $2 \mathrm{HDM}+\mathrm{S}$ type III (left) and type IV (right). The contour lines shown for $\mathcal{B}(\mathrm{h} \rightarrow \mathrm{aa})=1.0$ and 0.34 correspond to the colour scale indicated on the right vertical scale. The number 0.34 corresponds to the limit on the branching fraction of the Higgs boson to beyond-the-SM particles at 95\% CL obtained with data collected at center-of-mass energies of 7 and $8 \mathrm{TeV}$ by the CMS and ATLAS experiments [10].

\section{Acknowledgments}

We congratulate our colleagues in the CERN accelerator departments for the excellent performance of the LHC and thank the technical and administrative staffs at CERN and at other CMS institutes for their contributions to the success of the CMS effort. In addition, we gratefully acknowledge the computing centers and personnel of the Worldwide LHC Computing Grid for delivering so effectively the computing infrastructure essential to our analyses. Finally, we acknowledge the enduring support for the construction and operation of the LHC and the CMS detector provided by the following funding agencies: BMWFW and FWF (Austria); FNRS and FWO (Belgium); CNPq, CAPES, FAPERJ, and FAPESP (Brazil); MES (Bulgaria); CERN; CAS, MoST, and NSFC (China); COLCIENCIAS (Colombia); MSES and CSF (Croatia); RPF (Cyprus); SENESCYT (Ecuador); MoER, ERC IUT, and ERDF (Estonia); Academy of Finland, MEC, and HIP (Finland); CEA and CNRS/IN2P3 (France); BMBF, DFG, and HGF (Germany); GSRT (Greece); NKFIA (Hungary); DAE and DST (India); IPM (Iran); SFI (Ireland); INFN (Italy); MSIP and NRF (Republic of Korea); LAS (Lithuania); MOE and UM (Malaysia); BUAP, CINVESTAV, CONACYT, LNS, SEP, and UASLP-FAI (Mexico); MBIE (New Zealand); PAEC (Pakistan); MSHE and NSC (Poland); FCT (Portugal); JINR (Dubna); MON, RosAtom, RAS and RFBR (Russia); MESTD (Serbia); SEIDI, CPAN, PCTI and FEDER (Spain); Swiss Funding Agencies (Switzerland); MST (Taipei); ThEPCenter, IPST, STAR, and NSTDA (Thailand); TUBITAK and TAEK (Turkey); NASU and SFFR (Ukraine); STFC (United Kingdom); DOE and NSF (U.S.A.).

Individuals have received support from the Marie-Curie program and the European Research Council and Horizon 2020 Grant, contract No. 675440 (European Union); the 
Leventis Foundation; the A. P. Sloan Foundation; the Alexander von Humboldt Foundation; the Belgian Federal Science Policy Office; the Fonds pour la Formation à la Recherche dans l'Industrie et dans l'Agriculture (FRIA-Belgium); the Agentschap voor Innovatie door Wetenschap en Technologie (IWT-Belgium); the F.R.S.-FNRS and FWO (Belgium) under the "Excellence of Science - EOS" - be.h project n. 30820817; the Ministry of Education, Youth and Sports (MEYS) of the Czech Republic; the Lendület ("Momentum") Programme and the János Bolyai Research Scholarship of the Hungarian Academy of Sciences, the New National Excellence Program ÚNKP, the NKFIA research grants 123842, 123959, 124845, 124850 and 125105 (Hungary); the Council of Science and Industrial Research, India; the HOMING PLUS program of the Foundation for Polish Science, cofinanced from European Union, Regional Development Fund, the Mobility Plus program of the Ministry of Science and Higher Education, the National Science Center (Poland), contracts Harmonia 2014/14/M/ST2/00428, Opus 2014/13/B/ST2/02543, 2014/15/B/ST2/03998, and 2015/19/B/ST2/02861, Sonata-bis 2012/07/E/ST2/01406; the National Priorities Research Program by Qatar National Research Fund; the Programa Estatal de Fomento de la Investigación Científica y Técnica de Excelencia María de Maeztu, grant MDM-2015-0509 and the Programa Severo Ochoa del Principado de Asturias; the Thalis and Aristeia programs cofinanced by EU-ESF and the Greek NSRF; the Rachadapisek Sompot Fund for Postdoctoral Fellowship, Chulalongkorn University and the Chulalongkorn Academic into Its 2nd Century Project Advancement Project (Thailand); the Welch Foundation, contract C-1845; and the Weston Havens Foundation (U.S.A.).

Open Access. This article is distributed under the terms of the Creative Commons Attribution License (CC-BY 4.0), which permits any use, distribution and reproduction in any medium, provided the original author(s) and source are credited.

\section{References}

[1] ATLAS collaboration, Observation of a new particle in the search for the standard model Higgs boson with the ATLAS detector at the LHC, Phys. Lett. B 716 (2012) 1 [arXiv: 1207.7214] [inSPIRE].

[2] CMS collaboration, Observation of a new boson at a mass of $125 \mathrm{GeV}$ with the CMS experiment at the LHC, Phys. Lett. B 716 (2012) 30 [arXiv:1207.7235] [INSPIRE].

[3] CMS collaboration, Observation of a new boson with mass near $125 \mathrm{GeV}$ in pp collisions at $\sqrt{s}=7$ and $8 \mathrm{TeV}$, JHEP 06 (2013) 081 [arXiv: 1303.4571] [INSPIRE].

[4] F. Englert and R. Brout, Broken symmetry and the mass of gauge vector mesons, Phys. Rev. Lett. 13 (1964) 321 [INSPIRE].

[5] P.W. Higgs, Broken symmetries, massless particles and gauge fields, Phys. Lett. 12 (1964) 132 [INSPIRE].

[6] P.W. Higgs, Broken symmetries and the masses of gauge bosons, Phys. Rev. Lett. 13 (1964) 508 [INSPIRE].

[7] G.S. Guralnik, C.R. Hagen and T.W.B. Kibble, Global conservation laws and massless particles, Phys. Rev. Lett. 13 (1964) 585 [InSPIRE]. 
[8] P.W. Higgs, Spontaneous symmetry breakdown without massless bosons, Phys. Rev. 145 (1966) 1156 [INSPIRE].

[9] T.W.B. Kibble, Symmetry breaking in non-Abelian gauge theories, Phys. Rev. 155 (1967) 1554 [INSPIRE].

[10] ATLAS, CMS collaboration, Measurements of the Higgs boson production and decay rates and constraints on its couplings from a combined ATLAS and CMS analysis of the LHC pp collision data at $\sqrt{s}=7$ and $8 \mathrm{TeV}$, JHEP 08 (2016) 045 [arXiv:1606.02266] [INSPIRE].

[11] D. Curtin et al., Exotic decays of the 125 GeV Higgs boson, Phys. Rev. D 90 (2014) 075004 [arXiv: 1312.4992] [INSPIRE].

[12] U. Ellwanger, C. Hugonie and A.M. Teixeira, The next-to-minimal supersymmetric standard model, Phys. Rept. 496 (2010) 1 [arXiv:0910.1785] [InSPIRE].

[13] M. Maniatis, The next-to-minimal supersymmetric extension of the standard model reviewed, Int. J. Mod. Phys. A 25 (2010) 3505 [arXiv:0906.0777] [InSPIRE].

[14] CMS collaboration, Search for light bosons in decays of the $125 \mathrm{GeV}$ Higgs boson in proton-proton collisions at $\sqrt{s}=8 \mathrm{TeV}$, JHEP 10 (2017) 076 [arXiv:1701.02032] [INSPIRE].

[15] CMS collaboration, Search for a very light NMSSM Higgs boson produced in decays of the $125 \mathrm{GeV}$ scalar boson and decaying into $\tau$ leptons in pp collisions at $\sqrt{s}=8 \mathrm{TeV}$, JHEP 01 (2016) 079 [arXiv: 1510.06534] [INSPIRE].

[16] CMS collaboration, A search for pair production of new light bosons decaying into muons, Phys. Lett. B 752 (2016) 146 [arXiv: 1506.00424] [inSPIRE].

[17] ATLAS collaboration, Search for the Higgs boson produced in association with a $W$ boson and decaying to four b-quarks via two spin-zero particles in pp collisions at $13 \mathrm{TeV}$ with the ATLAS detector, Eur. Phys. J. C 76 (2016) 605 [arXiv: 1606.08391] [INSPIRE].

[18] ATLAS collaboration, Search for new light gauge bosons in Higgs boson decays to four-lepton final states in pp collisions at $\sqrt{s}=8 \mathrm{TeV}$ with the ATLAS detector at the LHC, Phys. Rev. D 92 (2015) 092001 [arXiv: 1505.07645] [INSPIRE].

[19] ATLAS collaboration, Search for new phenomena in events with at least three photons collected in pp collisions at $\sqrt{s}=8 \mathrm{TeV}$ with the ATLAS detector, Eur. Phys. J. C 76 (2016) 210 [arXiv: 1509.05051] [INSPIRE].

[20] ATLAS collaboration, Search for Higgs bosons decaying to aa in the $\mu \mu \tau \tau$ final state in pp collisions at $\sqrt{s}=8 \mathrm{TeV}$ with the ATLAS experiment, Phys. Rev. D 92 (2015) 052002 [arXiv: 1505.01609] [INSPIRE].

[21] CMS collaboration, The CMS trigger system, 2017 JINST 12 P01020 [arXiv:1609.02366] [INSPIRE].

[22] CMS collaboration, The CMS experiment at the CERN LHC, 2008 JINST 3 S08004 [INSPIRE].

[23] J. Alwall et al., The automated computation of tree-level and next-to-leading order differential cross sections and their matching to parton shower simulations, JHEP 07 (2014) 079 [arXiv: 1405.0301] [INSPIRE].

[24] J. Alwall et al., Comparative study of various algorithms for the merging of parton showers and matrix elements in hadronic collisions, Eur. Phys. J. C 53 (2008) 473 [arXiv:0706.2569] [INSPIRE].

[25] T. Sjöstrand et al., An introduction to PYTHIA 8.2, Comput. Phys. Commun. 191 (2015) 159 [arXiv: 1410.3012] [INSPIRE]. 
[26] CMS collaboration, Event generator tunes obtained from underlying event and multiparton scattering measurements, Eur. Phys. J. C 76 (2016) 155 [arXiv:1512.00815] [InSPIRE].

[27] S. Alioli, P. Nason, C. Oleari and E. Re, NLO vector-boson production matched with shower in POWHEG, JHEP 07 (2008) 060 [arXiv:0805.4802] [INSPIRE].

[28] P. Nason, A new method for combining NLO QCD with shower Monte Carlo algorithms, JHEP 11 (2004) 040 [hep-ph/0409146] [INSPIRE].

[29] S. Frixione, P. Nason and C. Oleari, Matching NLO QCD computations with Parton Shower simulations: the POWHEG method, JHEP 11 (2007) 070 [arXiv:0709.2092] [INSPIRE].

[30] J.M. Campbell and R.K. Ellis, MCFM for the Tevatron and the LHC, Nucl. Phys. Proc. Suppl. 205-206 (2010) 10 [arXiv: 1007.3492] [INSPIRE].

[31] NNPDF collaboration, R.D. Ball et al., Parton distributions for the LHC Run II, JHEP 04 (2015) 040 [arXiv: 1410.8849] [INSPIRE].

[32] M. Grazzini, S. Kallweit and D. Rathlev, ZZ production at the LHC: fiducial cross sections and distributions in NNLO QCD, Phys. Lett. B 750 (2015) 407 [arXiv:1507.06257] [INSPIRE].

[33] GEANT4 collaboration, S. Agostinelli et al., GEANT4: a simulation toolkit, Nucl. Instrum. Meth. A 506 (2003) 250 [InSPIRE].

[34] CMS collaboration, Particle-flow reconstruction and global event description with the CMS detector, 2017 JINST 12 P10003 [arXiv:1706.04965] [INSPIRE].

[35] M. Cacciari, G.P. Salam and G. Soyez, The anti- $k_{t}$ jet clustering algorithm, JHEP 04 (2008) 063 [arXiv:0802.1189] [INSPIRE].

[36] M. Cacciari, G.P. Salam and G. Soyez, FastJet user manual, Eur. Phys. J. C 72 (2012) 1896 [arXiv:1111.6097] [INSPIRE].

[37] CMS collaboration, Performance of electron reconstruction and selection with the CMS detector in proton-proton collisions at $\sqrt{s}=8 \mathrm{TeV}, 2015$ JINST $10 \mathrm{P} 06005$ [arXiv: 1502.02701] [INSPIRE].

[38] CMS collaboration, Performance of CMS muon reconstruction in pp collision events at $\sqrt{s}=7$ TeV, 2012 JINST 7 P10002 [arXiv:1206.4071] [INSPIRE].

[39] M. Cacciari and G.P. Salam, Dispelling the $N^{3}$ myth for the $k_{t}$ jet-finder, Phys. Lett. B 641 (2006) 57 [hep-ph/0512210] [INSPIRE].

[40] CMS collaboration, Identification of heavy-flavour jets with the CMS detector in pp collisions at $13 \mathrm{TeV}, 2018$ JINST 13 P05011 [arXiv: 1712.07158] [INSPIRE].

[41] CMS collaboration, Reconstruction and identification of $\tau$ lepton decays to hadrons and $\nu_{\tau}$ at CMS, 2016 JINST 11 P01019 [arXiv:1510.07488] [INSPIRE].

[42] CMS collaboration, Performance of reconstruction and identification of tau leptons in their decays to hadrons and tau neutrino in LHC Run-2, CMS-PAS-TAU-16-002 (2016).

[43] R.A. Fisher, On the interpretation of $\chi^{2}$ from contingency tables, and the calculation of $p$, J. Roy. Stat. Soc. 85 (1922) 87.

[44] CMS collaboration, CMS luminosity measurements for the 2016 data taking period, CMS-PAS-LUM-17-001 (2017).

[45] ATLAS and CMS collaborations and the LHC Higgs Combination Group, Procedure for the LHC Higgs boson search combination in Summer 2011, CMS-NOTE-2011-005 (2011).

[46] CMS collaboration, Combined results of searches for the standard model Higgs boson in pp collisions at $\sqrt{s}=7$ TeV, Phys. Lett. B 710 (2012) 26 [arXiv:1202.1488] [INSPIRE]. 
[47] T. Junk, Confidence level computation for combining searches with small statistics, Nucl. Instrum. Meth. A 434 (1999) 435 [hep-ex/9902006] [INSPIRE].

[48] A.L. Read, Presentation of search results: the $C L_{s}$ technique, J. Phys. G 28 (2002) 2693 [INSPIRE].

[49] A. Djouadi, The anatomy of electro-weak symmetry breaking. I: the Higgs boson in the standard model, Phys. Rept. 457 (2008) 1 [hep-ph/0503172] [INSPIRE]. 


\section{The CMS collaboration}

\section{Yerevan Physics Institute, Yerevan, Armenia}

A.M. Sirunyan, A. Tumasyan

\section{Institut für Hochenergiephysik, Wien, Austria}

W. Adam, F. Ambrogi, E. Asilar, T. Bergauer, J. Brandstetter, M. Dragicevic, J. Erö, A. Escalante Del Valle, M. Flechl, R. Frühwirth ${ }^{1}$, V.M. Ghete, J. Hrubec, M. Jeitler ${ }^{1}$, N. Krammer, I. Krätschmer, D. Liko, T. Madlener, I. Mikulec, N. Rad, H. Rohringer, J. Schieck ${ }^{1}$, R. Schöfbeck, M. Spanring, D. Spitzbart, A. Taurok, W. Waltenberger, J. Wittmann, C.-E. Wulz ${ }^{1}$, M. Zarucki

\section{Institute for Nuclear Problems, Minsk, Belarus}

V. Chekhovsky, V. Mossolov, J. Suarez Gonzalez

\section{Universiteit Antwerpen, Antwerpen, Belgium}

E.A. De Wolf, D. Di Croce, X. Janssen, J. Lauwers, M. Pieters, M. Van De Klundert, H. Van Haevermaet, P. Van Mechelen, N. Van Remortel

\section{Vrije Universiteit Brussel, Brussel, Belgium}

S. Abu Zeid, F. Blekman, J. D'Hondt, I. De Bruyn, J. De Clercq, K. Deroover, G. Flouris, D. Lontkovskyi, S. Lowette, I. Marchesini, S. Moortgat, L. Moreels, Q. Python, K. Skovpen, S. Tavernier, W. Van Doninck, P. Van Mulders, I. Van Parijs

\section{Université Libre de Bruxelles, Bruxelles, Belgium}

D. Beghin, B. Bilin, H. Brun, B. Clerbaux, G. De Lentdecker, H. Delannoy, B. Dorney, G. Fasanella, L. Favart, R. Goldouzian, A. Grebenyuk, A.K. Kalsi, T. Lenzi, J. Luetic, N. Postiau, E. Starling, L. Thomas, C. Vander Velde, P. Vanlaer, D. Vannerom, Q. Wang

\section{Ghent University, Ghent, Belgium}

T. Cornelis, D. Dobur, A. Fagot, M. Gul, I. Khvastunov², D. Poyraz, C. Roskas, D. Trocino, M. Tytgat, W. Verbeke, B. Vermassen, M. Vit, N. Zaganidis

\section{Université Catholique de Louvain, Louvain-la-Neuve, Belgium}

H. Bakhshiansohi, O. Bondu, S. Brochet, G. Bruno, C. Caputo, P. David, C. Delaere, M. Delcourt, B. Francois, A. Giammanco, G. Krintiras, V. Lemaitre, A. Magitteri, A. Mertens, M. Musich, K. Piotrzkowski, A. Saggio, M. Vidal Marono, S. Wertz, J. Zobec

\section{Centro Brasileiro de Pesquisas Fisicas, Rio de Janeiro, Brazil}

F.L. Alves, G.A. Alves, L. Brito, M. Correa Martins Junior, G. Correia Silva, C. Hensel, A. Moraes, M.E. Pol, P. Rebello Teles

\section{Universidade do Estado do Rio de Janeiro, Rio de Janeiro, Brazil}

E. Belchior Batista Das Chagas, W. Carvalho, J. Chinellato ${ }^{3}$, E. Coelho, E.M. Da Costa, G.G. Da Silveira ${ }^{4}$, D. De Jesus Damiao, C. De Oliveira Martins, S. Fonseca De Souza, H. Malbouisson, D. Matos Figueiredo, M. Melo De Almeida, C. Mora Herrera, L. Mundim, H. Nogima, W.L. Prado Da Silva, L.J. Sanchez Rosas, A. Santoro, A. Sznajder, M. Thiel, E.J. Tonelli Manganote ${ }^{3}$, F. Torres Da Silva De Araujo, A. Vilela Pereira 
Universidade Estadual Paulista ${ }^{a}$, Universidade Federal do $\mathbf{A B C}^{b}$, São Paulo, Brazil

S. Ahuja ${ }^{a}$, C.A. Bernardes ${ }^{a}$, L. Calligaris ${ }^{a}$, T.R. Fernandez Perez Tomei ${ }^{a}$, E.M. Gregores ${ }^{b}$, P.G. Mercadante ${ }^{b}$, S.F. Novaes ${ }^{a}$, SandraS. Padula $^{a}$, D. Romero Abad ${ }^{b}$

Institute for Nuclear Research and Nuclear Energy, Bulgarian Academy of Sciences, Sofia, Bulgaria

A. Aleksandrov, R. Hadjiiska, P. Iaydjiev, A. Marinov, M. Misheva, M. Rodozov,

M. Shopova, G. Sultanov

University of Sofia, Sofia, Bulgaria

A. Dimitrov, L. Litov, B. Pavlov, P. Petkov

Beihang University, Beijing, China

W. Fang ${ }^{5}$, X. Gao ${ }^{5}$, L. Yuan

Institute of High Energy Physics, Beijing, China

M. Ahmad, J.G. Bian, G.M. Chen, H.S. Chen, M. Chen, Y. Chen, C.H. Jiang, D. Leggat, H. Liao, Z. Liu, F. Romeo, S.M. Shaheen ${ }^{6}$, A. Spiezia, J. Tao, C. Wang, Z. Wang, E. Yazgan,

H. Zhang, J. Zhao

State Key Laboratory of Nuclear Physics and Technology, Peking University, Beijing, China

Y. Ban, G. Chen, A. Levin, J. Li, L. Li, Q. Li, Y. Mao, S.J. Qian, D. Wang, Z. Xu

Tsinghua University, Beijing, China

Y. Wang

Universidad de Los Andes, Bogota, Colombia

C. Avila, A. Cabrera, C.A. Carrillo Montoya, L.F. Chaparro Sierra, C. Florez,

C.F. González Hernández, M.A. Segura Delgado

University of Split, Faculty of Electrical Engineering, Mechanical Engineering and Naval Architecture, Split, Croatia

B. Courbon, N. Godinovic, D. Lelas, I. Puljak, T. Sculac

University of Split, Faculty of Science, Split, Croatia

Z. Antunovic, M. Kovac

Institute Rudjer Boskovic, Zagreb, Croatia

V. Brigljevic, D. Ferencek, K. Kadija, B. Mesic, A. Starodumov ${ }^{7}$, T. Susa

University of Cyprus, Nicosia, Cyprus

M.W. Ather, A. Attikis, E. Erodotou, M. Kolosova, G. Mavromanolakis, J. Mousa,

C. Nicolaou, F. Ptochos, P.A. Razis, H. Rykaczewski

Charles University, Prague, Czech Republic

M. Finger ${ }^{8}$, M. Finger Jr. ${ }^{8}$ 
Escuela Politecnica Nacional, Quito, Ecuador

E. Ayala

Universidad San Francisco de Quito, Quito, Ecuador

E. Carrera Jarrin

Academy of Scientific Research and Technology of the Arab Republic of Egypt, Egyptian Network of High Energy Physics, Cairo, Egypt

H. Abdalla ${ }^{9}$, A.A. Abdelalim ${ }^{10,11}$, A. Mohamed $^{11}$

National Institute of Chemical Physics and Biophysics, Tallinn, Estonia

S. Bhowmik, A. Carvalho Antunes De Oliveira, R.K. Dewanjee, K. Ehataht, M. Kadastik, M. Raidal, C. Veelken

Department of Physics, University of Helsinki, Helsinki, Finland

P. Eerola, H. Kirschenmann, J. Pekkanen, M. Voutilainen

Helsinki Institute of Physics, Helsinki, Finland

J. Havukainen, J.K. Heikkilä, T. Järvinen, V. Karimäki, R. Kinnunen, T. Lampén, K. Lassila-Perini, S. Laurila, S. Lehti, T. Lindén, P. Luukka, T. Mäenpää, H. Siikonen, E. Tuominen, J. Tuominiemi

Lappeenranta University of Technology, Lappeenranta, Finland

T. Tuuva

IRFU, CEA, Université Paris-Saclay, Gif-sur-Yvette, France

M. Besancon, F. Couderc, M. Dejardin, D. Denegri, J.L. Faure, F. Ferri, S. Ganjour,

A. Givernaud, P. Gras, G. Hamel de Monchenault, P. Jarry, C. Leloup, E. Locci, J. Malcles,

G. Negro, J. Rander, A. Rosowsky, M.Ö. Sahin, M. Titov

Laboratoire Leprince-Ringuet, Ecole polytechnique, CNRS/IN2P3, Université Paris-Saclay, Palaiseau, France

A. Abdulsalam ${ }^{12}$, C. Amendola, I. Antropov, F. Beaudette, P. Busson, C. Charlot,

R. Granier de Cassagnac, I. Kucher, S. Lisniak, A. Lobanov, J. Martin Blanco, M. Nguyen,

C. Ochando, G. Ortona, P. Pigard, R. Salerno, J.B. Sauvan, Y. Sirois, A.G. Stahl Leiton,

A. Zabi, A. Zghiche

Université de Strasbourg, CNRS, IPHC UMR 7178, F-67000 Strasbourg, France

J.-L. Agram ${ }^{13}$, J. Andrea, D. Bloch, J.-M. Brom, E.C. Chabert, V. Cherepanov, C. Collard, E. Conte ${ }^{13}$, J.-C. Fontaine ${ }^{13}$, D. Gelé, U. Goerlach, M. Jansová, A.-C. Le Bihan, N. Tonon, P. Van Hove

Centre de Calcul de l'Institut National de Physique Nucleaire et de Physique des Particules, CNRS/IN2P3, Villeurbanne, France

S. Gadrat 
Université de Lyon, Université Claude Bernard Lyon 1, CNRS-IN2P3, Institut de Physique Nucléaire de Lyon, Villeurbanne, France

S. Beauceron, C. Bernet, G. Boudoul, N. Chanon, R. Chierici, D. Contardo, P. Depasse, H. El Mamouni, J. Fay, L. Finco, S. Gascon, M. Gouzevitch, G. Grenier, B. Ille, F. Lagarde, I.B. Laktineh, H. Lattaud, M. Lethuillier, L. Mirabito, A.L. Pequegnot, S. Perries, A. Popov ${ }^{14}$, V. Sordini, M. Vander Donckt, S. Viret, S. Zhang

\section{Georgian Technical University, Tbilisi, Georgia}

A. Khvedelidze ${ }^{8}$

Tbilisi State University, Tbilisi, Georgia

Z. Tsamalaidze ${ }^{8}$

RWTH Aachen University, I. Physikalisches Institut, Aachen, Germany

C. Autermann, L. Feld, M.K. Kiesel, K. Klein, M. Lipinski, M. Preuten, M.P. Rauch, C. Schomakers, J. Schulz, M. Teroerde, B. Wittmer, V. Zhukov ${ }^{14}$

RWTH Aachen University, III. Physikalisches Institut A, Aachen, Germany

A. Albert, D. Duchardt, M. Endres, M. Erdmann, T. Esch, R. Fischer, S. Ghosh, A. Güth, T. Hebbeker, C. Heidemann, K. Hoepfner, H. Keller, S. Knutzen, L. Mastrolorenzo, M. Merschmeyer, A. Meyer, P. Millet, S. Mukherjee, T. Pook, M. Radziej, H. Reithler, M. Rieger, F. Scheuch, A. Schmidt, D. Teyssier

RWTH Aachen University, III. Physikalisches Institut B, Aachen, Germany

G. Flügge, O. Hlushchenko, B. Kargoll, T. Kress, A. Künsken, T. Müller, A. Nehrkorn, A. Nowack, C. Pistone, O. Pooth, H. Sert, A. Stahl ${ }^{15}$

\section{Deutsches Elektronen-Synchrotron, Hamburg, Germany}

M. Aldaya Martin, T. Arndt, C. Asawatangtrakuldee, I. Babounikau, K. Beernaert, O. Behnke, U. Behrens, A. Bermúdez Martínez, D. Bertsche, A.A. Bin Anuar, K. Borras ${ }^{16}$, V. Botta, A. Campbell, P. Connor, C. Contreras-Campana, F. Costanza, V. Danilov, A. De Wit, M.M. Defranchis, C. Diez Pardos, D. Domínguez Damiani, G. Eckerlin, T. Eichhorn, A. Elwood, E. Eren, E. Gallo ${ }^{17}$, A. Geiser, J.M. Grados Luyando, A. Grohsjean, P. Gunnellini, M. Guthoff, M. Haranko, A. Harb, J. Hauk, H. Jung, M. Kasemann, J. Keaveney, C. Kleinwort, J. Knolle, D. Krücker, W. Lange, A. Lelek, T. Lenz, K. Lipka, W. Lohmann ${ }^{18}$, R. Mankel, I.-A. Melzer-Pellmann, A.B. Meyer, M. Meyer, M. Missiroli, G. Mittag, J. Mnich, V. Myronenko, S.K. Pflitsch, D. Pitzl, A. Raspereza, M. Savitskyi, P. Saxena, P. Schütze, C. Schwanenberger, R. Shevchenko, A. Singh, N. Stefaniuk, H. Tholen, O. Turkot, A. Vagnerini, G.P. Van Onsem, R. Walsh, Y. Wen, K. Wichmann, C. Wissing, O. Zenaiev

\section{University of Hamburg, Hamburg, Germany}

R. Aggleton, S. Bein, L. Benato, A. Benecke, V. Blobel, M. Centis Vignali, T. Dreyer, E. Garutti, D. Gonzalez, J. Haller, A. Hinzmann, A. Karavdina, G. Kasieczka, R. Klanner, R. Kogler, N. Kovalchuk, S. Kurz, V. Kutzner, J. Lange, D. Marconi, J. Multhaup, M. Niedziela, D. Nowatschin, A. Perieanu, A. Reimers, O. Rieger, C. Scharf, P. Schleper, 
S. Schumann, J. Schwandt, J. Sonneveld, H. Stadie, G. Steinbrück, F.M. Stober, M. Stöver, D. Troendle, A. Vanhoefer, B. Vormwald

Institut für Experimentelle Teilchenphysik, Karlsruhe, Germany

M. Akbiyik, C. Barth, M. Baselga, S. Baur, E. Butz, R. Caspart, T. Chwalek, F. Colombo, W. De Boer, A. Dierlamm, N. Faltermann, B. Freund, M. Giffels, M.A. Harrendorf, F. Hartmann ${ }^{15}$, S.M. Heindl, U. Husemann, F. Kassel ${ }^{15}$, I. Katkov ${ }^{14}$, S. Kudella, H. Mildner, S. Mitra, M.U. Mozer, Th. Müller, M. Plagge, G. Quast, K. Rabbertz, M. Schröder, I. Shvetsov, G. Sieber, H.J. Simonis, R. Ulrich, S. Wayand, M. Weber, T. Weiler, S. Williamson, C. Wöhrmann, R. Wolf

Institute of Nuclear and Particle Physics (INPP), NCSR Demokritos, Aghia Paraskevi, Greece

G. Anagnostou, G. Daskalakis, T. Geralis, A. Kyriakis, D. Loukas, G. Paspalaki, I. TopsisGiotis

National and Kapodistrian University of Athens, Athens, Greece

G. Karathanasis, S. Kesisoglou, P. Kontaxakis, A. Panagiotou, N. Saoulidou, E. Tziaferi, K. Vellidis

National Technical University of Athens, Athens, Greece

K. Kousouris, I. Papakrivopoulos, G. Tsipolitis

University of Ioánnina, Ioánnina, Greece

I. Evangelou, C. Foudas, P. Gianneios, P. Katsoulis, P. Kokkas, S. Mallios, N. Manthos, I. Papadopoulos, E. Paradas, J. Strologas, F.A. Triantis, D. Tsitsonis

MTA-ELTE Lendület CMS Particle and Nuclear Physics Group, Eötvös Loránd University, Budapest, Hungary

M. Bartók ${ }^{19}$, M. Csanad, N. Filipovic, P. Major, M.I. Nagy, G. Pasztor, O. Surányi, G.I. Veres

Wigner Research Centre for Physics, Budapest, Hungary

G. Bencze, C. Hajdu, D. Horvath ${ }^{20}$, Á. Hunyadi, F. Sikler, T.Á. Vámi, V. Veszpremi, G. Vesztergombi ${ }^{\dagger}$

Institute of Nuclear Research ATOMKI, Debrecen, Hungary

N. Beni, S. Czellar, J. Karancsi ${ }^{21}$, A. Makovec, J. Molnar, Z. Szillasi

Institute of Physics, University of Debrecen, Debrecen, Hungary

P. Raics, Z.L. Trocsanyi, B. Ujvari

Indian Institute of Science (IISc), Bangalore, India

S. Choudhury, J.R. Komaragiri, P.C. Tiwari

National Institute of Science Education and Research, HBNI, Bhubaneswar, India

S. Bahinipati22 , C. Kar, P. Mal, K. Mandal, A. $\operatorname{Nayak}^{23}$, D.K. Sahoo ${ }^{22}$, S.K. Swain 
Panjab University, Chandigarh, India

S. Bansal, S.B. Beri, V. Bhatnagar, S. Chauhan, R. Chawla, N. Dhingra, R. Gupta,

A. Kaur, A. Kaur, M. Kaur, S. Kaur, R. Kumar, P. Kumari, M. Lohan, A. Mehta,

K. Sandeep, S. Sharma, J.B. Singh, G. Walia

University of Delhi, Delhi, India

A. Bhardwaj, B.C. Choudhary, R.B. Garg, M. Gola, S. Keshri, Ashok Kumar, S. Malhotra, M. Naimuddin, P. Priyanka, K. Ranjan, Aashaq Shah, R. Sharma

Saha Institute of Nuclear Physics, HBNI, Kolkata, India

R. Bhardwaj ${ }^{24}$, M. Bharti, R. Bhattacharya, S. Bhattacharya, U. Bhawandeep ${ }^{24}$, D. Bhowmik, S. Dey, S. Dutt ${ }^{24}$, S. Dutta, S. Ghosh, K. Mondal, S. Nandan, A. Purohit, P.K. Rout, A. Roy, S. Roy Chowdhury, S. Sarkar, M. Sharan, B. Singh, S. Thakur ${ }^{24}$

Indian Institute of Technology Madras, Madras, India

P.K. Behera

Bhabha Atomic Research Centre, Mumbai, India

R. Chudasama, D. Dutta, V. Jha, V. Kumar, P.K. Netrakanti, L.M. Pant, P. Shukla

Tata Institute of Fundamental Research-A, Mumbai, India

T. Aziz, M.A. Bhat, S. Dugad, G.B. Mohanty, N. Sur, B. Sutar, RavindraKumar Verma

Tata Institute of Fundamental Research-B, Mumbai, India

S. Banerjee, S. Bhattacharya, S. Chatterjee, P. Das, M. Guchait, Sa. Jain, S. Karmakar, S. Kumar, M. Maity ${ }^{25}$, G. Majumder, K. Mazumdar, N. Sahoo, T. Sarkar ${ }^{25}$

Indian Institute of Science Education and Research (IISER), Pune, India

S. Chauhan, S. Dube, V. Hegde, A. Kapoor, K. Kothekar, S. Pandey, A. Rane, S. Sharma

Institute for Research in Fundamental Sciences (IPM), Tehran, Iran

S. Chenarani ${ }^{26}$, E. Eskandari Tadavani, S.M. Etesami ${ }^{26}$, M. Khakzad, M. Mohammadi Najafabadi, M. Naseri, F. Rezaei Hosseinabadi, B. Safarzadeh ${ }^{27}$, M. Zeinali

University College Dublin, Dublin, Ireland

M. Felcini, M. Grunewald

INFN Sezione di Bari ${ }^{a}$, Università di $\operatorname{Bari}^{b}$, Politecnico di Bari ${ }^{c}$, Bari, Italy

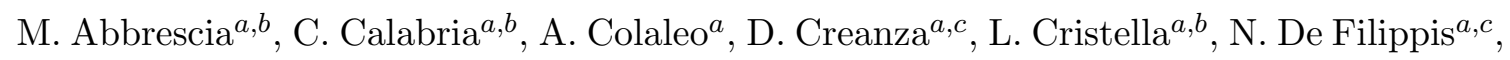
M. De Palma ${ }^{a, b}$, A. Di Florio ${ }^{a, b}$, F. Errico ${ }^{a, b}$, L. Fiore $^{a}$, A. Gelmi ${ }^{a, b}$, G. Iaselli $^{a, c}$, M. Ince $^{a, b}$, S. Lezki ${ }^{a, b}$, G. Maggi ${ }^{a, c}$, M. Maggi ${ }^{a}$, G. Miniello ${ }^{a, b}, \mathrm{~S} . \mathrm{My}^{a, b}, \mathrm{~S} . \mathrm{Nuzzo}^{a, b}$, A. Pompilia ${ }^{a, b}$, G. Pugliese ${ }^{a, c}$, R. Radogna ${ }^{a}$, A. Ranieri ${ }^{a}$, G. Selvaggi ${ }^{a, b}$, A. Sharma ${ }^{a}$, L. Silvestris ${ }^{a}$, R. Venditti ${ }^{a}$, P. Verwilligen ${ }^{a}$, G. Zito ${ }^{a}$

INFN Sezione di Bologna ${ }^{a}$, Università di Bologna ${ }^{b}$, Bologna, Italy

G. Abbiendi ${ }^{a}$, C. Battilana ${ }^{a, b}$, D. Bonacorsi ${ }^{a, b}$, L. Borgonovi $^{a, b}$, S. Braibant-Giacomelli ${ }^{a, b}$,

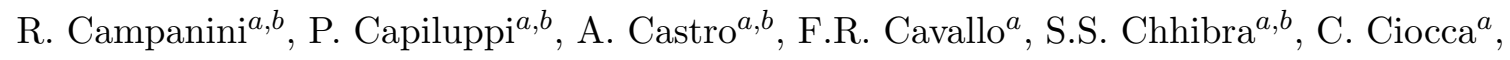

G. Codispoti ${ }^{a, b}$, M. Cuffiani ${ }^{a, b}$, G.M. Dallavalle ${ }^{a}$, F. Fabbri $^{a}$, A. Fanfani ${ }^{a, b}$, P. Giacomelli ${ }^{a}$,

C. Grandi ${ }^{a}$, L. Guiducci ${ }^{a, b}$, F. Iemmi ${ }^{a, b}$, S. Marcellini ${ }^{a}$, G. Masetti ${ }^{a}$, A. Montanari ${ }^{a}$, 


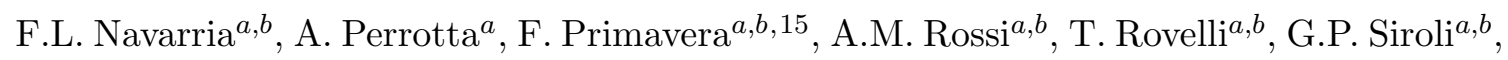
N. $\operatorname{Tosi}^{a}$

INFN Sezione di Catania ${ }^{a}$, Università di Catania ${ }^{b}$, Catania, Italy

S. Albergo ${ }^{a, b}$, A. Di Mattia ${ }^{a}$, R. Potenza ${ }^{a, b}$, A. Tricomi ${ }^{a, b}$, C. Tuve $^{a, b}$

INFN Sezione di Firenze ${ }^{a}$, Università di Firenze ${ }^{b}$, Firenze, Italy

G. Barbagli ${ }^{a}$, K. Chatterjee ${ }^{a, b}$, V. Ciulli ${ }^{a, b}$, C. Civinini ${ }^{a}$, R. D'Alessandro ${ }^{a, b}$, E. Focardi $^{a, b}{ }^{,}$

G. Latino, P. Lenzi ${ }^{a, b}$, M. Meschini ${ }^{a}$, S. Paoletti ${ }^{a}$, L. Russo $^{a}, 28$, G. Sguazzoni ${ }^{a}$, D. Strom $^{a}$,

L. Viliani ${ }^{a}$

INFN Laboratori Nazionali di Frascati, Frascati, Italy

L. Benussi, S. Bianco, F. Fabbri, D. Piccolo

INFN Sezione di Genova ${ }^{a}$, Università di Genova ${ }^{b}$, Genova, Italy

F. Ferro ${ }^{a}$, F. Ravera ${ }^{a, b}$, E. Robutti ${ }^{a}$, S. Tosi ${ }^{a, b}$

INFN Sezione di Milano-Bicocca ${ }^{a}$, Università di Milano-Bicocca ${ }^{b}$, Milano, Italy

A. Benaglia ${ }^{a}$, A. Beschi ${ }^{b}$, L. Brianza ${ }^{a, b}$, F. Brivio ${ }^{a, b}$, V. Ciriolo ${ }^{a, b, 15}$, S. Di Guida ${ }^{a, d, 15}$, M.E. Dinardo ${ }^{a, b}$, S. Fiorendi ${ }^{a, b}$, S. Gennai ${ }^{a}$, A. Ghezzi ${ }^{a, b}$, P. Govoni ${ }^{a, b}$, M. Malberti ${ }^{a, b}$, S. Malvezzi ${ }^{a}$, A. Massironi ${ }^{a, b}$, D. Menasce ${ }^{a}$, L. Moroni ${ }^{a}$, M. Paganoni ${ }^{a}, b$, D. Pedrini ${ }^{a}$, S. Ragazzi ${ }^{a, b}$, T. Tabarelli de Fatis ${ }^{a, b}$

INFN Sezione di Napoli ${ }^{a}$, Università di Napoli 'Federico II' ${ }^{b}$, Napoli, Italy, Università della Basilicata $^{c}$, Potenza, Italy, Università G. Marconi ${ }^{d}$, Roma, Italy

S. Buontempo ${ }^{a}$, N. Cavallo ${ }^{a, c}$, A. Di Crescenzo ${ }^{a, b}$, F. Fabozzi ${ }^{a, c}$, F. Fienga ${ }^{a}$, G. Galati ${ }^{a}$,

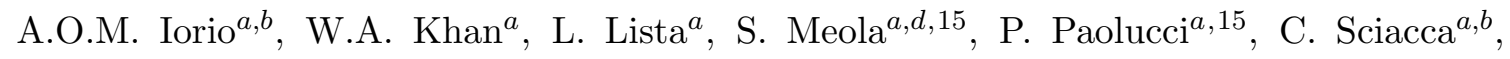
E. Voevodina ${ }^{a, b}$

INFN Sezione di Padova ${ }^{a}$, Università di Padova ${ }^{b}$, Padova, Italy, Università di Trento $^{c}$, Trento, Italy

P. Azzi ${ }^{a}$, N. Bacchetta ${ }^{a}$, D. Bisello ${ }^{a, b}$, A. Boletti ${ }^{a, b}$, A. Bragagnolo, R. Carlin ${ }^{a, b}$, P. Checchia ${ }^{a}$, M. Dall'Osso ${ }^{a, b}$, P. De Castro Manzano ${ }^{a}$, T. Dorigo ${ }^{a}$, U. Dosselli ${ }^{a}$, F. Gasparini ${ }^{a, b}$, U. Gasparini ${ }^{a, b}$, S. Lacaprara ${ }^{a}$, P. Lujan, M. Margoni ${ }^{a, b}$, A.T. Meneguzzo ${ }^{a, b}$, J. Pazzini ${ }^{a, b}$, N. Pozzobon ${ }^{a, b}$, P. Ronchese ${ }^{a, b}$, R. Rossin ${ }^{a, b}$, F. Simonetto ${ }^{a, b}$, A. Tiko, E. Torassa ${ }^{a}$, S. Ventura ${ }^{a}$, M. Zanetti ${ }^{a, b}$, P. Zotto ${ }^{a, b}$

INFN Sezione di Pavia ${ }^{a}$, Università di Pavia ${ }^{b}$, Pavia, Italy

A. Braghieri ${ }^{a}$, A. Magnani ${ }^{a}$, P. Montagna ${ }^{a, b}$, S.P. Ratti ${ }^{a, b}$, V. $\operatorname{Re}^{a}$, M. Ressegotti $^{a, b}$, C. Riccardi ${ }^{a, b}$, P. Salvini ${ }^{a}$, I. Vai ${ }^{a, b}$, P. Vitulo ${ }^{a, b}$

INFN Sezione di Perugia ${ }^{a}$, Università di Perugia ${ }^{b}$, Perugia, Italy

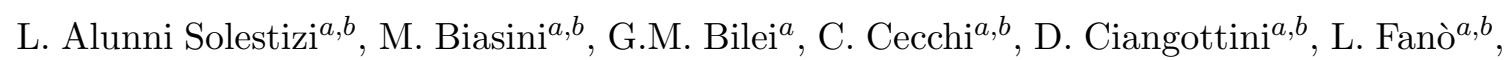
P. Lariccia ${ }^{a, b}$, R. Leonardi ${ }^{a, b}$, E. Manoni $^{a}$, G. Mantovani ${ }^{a, b}$, V. Mariani $^{a}{ }^{a}$, M. Menichelli $^{a}$, A. Rossi ${ }^{a, b}$, A. Santocchia ${ }^{a, b}$, D. Spiga ${ }^{a}$ 
INFN Sezione di Pisa ${ }^{a}$, Università di $\mathrm{Pisa}^{b}$, Scuola Normale Superiore di Pisa ${ }^{c}$, Pisa, Italy

K. Androsov ${ }^{a}$, P. Azzurri ${ }^{a}$, G. Bagliesi ${ }^{a}$, L. Bianchini ${ }^{a}$, T. Boccali ${ }^{a}$, L. Borrello, R. Castaldi ${ }^{a}$, M.A. Ciocci ${ }^{a, b}$, R. Dell'Orso ${ }^{a}$, G. Fedi ${ }^{a}$, F. Fiori ${ }^{a, c}$, L. Giannini $^{a, c}$, A. Giassi $^{a}$, M.T. Grippo ${ }^{a}$, F. Ligabue ${ }^{a, c}$, E. Manca ${ }^{a, c}$, G. Mandorli ${ }^{a, c}$, A. Messineo ${ }^{a, b}$, F. Palla ${ }^{a}$, A. Rizzi ${ }^{a, b}$, P. Spagnolo ${ }^{a}$, R. Tenchini ${ }^{a}$, G. Tonelli ${ }^{a}, b$ A. Venturi ${ }^{a}$, P.G. Verdini ${ }^{a}$

INFN Sezione di Roma ${ }^{a}$, Sapienza Università di Roma ${ }^{b}$, Rome, Italy

L. Barone ${ }^{a, b}$, F. Cavallari $^{a}$, M. Cipriani ${ }^{a, b}$, N. Daci ${ }^{a}$, D. Del Re ${ }^{a, b}$, E. Di Marco ${ }^{a, b}$, M. Diemoz ${ }^{a}$, S. Gelli ${ }^{a, b}$, E. Longo ${ }^{a, b}$, B. Marzocchi ${ }^{a, b}$, P. Meridiani ${ }^{a}$, G. Organtini ${ }^{a, b}$,

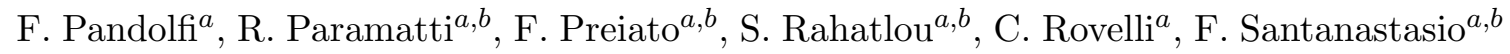

INFN Sezione di Torino ${ }^{a}$, Università di Torino ${ }^{b}$, Torino, Italy, Università del Piemonte Orientale ${ }^{c}$, Novara, Italy

N. Amapane ${ }^{a, b}$, R. Arcidiacono ${ }^{a, c}$, S. Argiro ${ }^{a, b}$, M. Arneodo $^{a, c}$, N. Bartosik ${ }^{a}$, R. Bellan $^{a, b}$, C. Biino ${ }^{a}$, N. Cartiglia $a$, F. Cenna ${ }^{a, b}$, S. Cometti, M. Costa ${ }^{a, b}$, R. Covarellia ${ }^{a, b}$, N. Demaria ${ }^{a}$, B. Kiani ${ }^{a, b}$, C. Mariotti ${ }^{a}$, S. Maselli ${ }^{a}$, E. Migliore ${ }^{a, b}$, V. Monaco ${ }^{a, b}$, E. Monteil ${ }^{a, b}$, M. Monteno ${ }^{a}$, M.M. Obertino ${ }^{a}, b$, L. Pacher ${ }^{a}, b$, N. Pastrone ${ }^{a}$, M. Pelliccioni ${ }^{a}$, G.L. Pinna Angioni ${ }^{a, b}$, A. Romero ${ }^{a, b}$, M. Ruspa ${ }^{a, c}$, R. Sacchi ${ }^{a, b}$, K. Shchelina ${ }^{a, b}$, V. Sola $^{a}$, A. Solano ${ }^{a, b}$, D. Soldi, A. Staiano ${ }^{a}$

INFN Sezione di Trieste ${ }^{a}$, Università di Trieste ${ }^{b}$, Trieste, Italy

S. Belforte ${ }^{a}$, V. Candelise ${ }^{a, b}$, M. Casarsa ${ }^{a}$, F. Cossutti ${ }^{a}$, G. Della Ricca ${ }^{a, b}$, F. Vazzoler $^{a, b}$, A. Zanetti ${ }^{a}$

Kyungpook National University

D.H. Kim, G.N. Kim, M.S. Kim, J. Lee, S. Lee, S.W. Lee, C.S. Moon, Y.D. Oh, S. Sekmen, D.C. Son, Y.C. Yang

Chonnam National University, Institute for Universe and Elementary Particles, Kwangju, Korea

H. Kim, D.H. Moon, G. Oh

Hanyang University, Seoul, Korea

J. Goh ${ }^{29}$, T.J. Kim

Korea University, Seoul, Korea

S. Cho, S. Choi, Y. Go, D. Gyun, S. Ha, B. Hong, Y. Jo, K. Lee, K.S. Lee, S. Lee, J. Lim, S.K. Park, Y. Roh

Sejong University, Seoul, Korea

H.S. Kim

Seoul National University, Seoul, Korea

J. Almond, J. Kim, J.S. Kim, H. Lee, K. Lee, K. Nam, S.B. Oh, B.C. Radburn-Smith, S.h. Seo, U.K. Yang, H.D. Yoo, G.B. Yu

University of Seoul, Seoul, Korea

D. Jeon, H. Kim, J.H. Kim, J.S.H. Lee, I.C. Park 
Sungkyunkwan University, Suwon, Korea

Y. Choi, C. Hwang, J. Lee, I. Yu

Vilnius University, Vilnius, Lithuania

V. Dudenas, A. Juodagalvis, J. Vaitkus

National Centre for Particle Physics, Universiti Malaya, Kuala Lumpur, Malaysia

I. Ahmed, Z.A. Ibrahim, M.A.B. Md $\mathrm{Ali}^{30}$, F. Mohamad Idris ${ }^{31}$, W.A.T. Wan Abdullah, M.N. Yusli, Z. Zolkapli

Universidad de Sonora (UNISON), Hermosillo, Mexico

A. Castaneda Hernandez, J.A. Murillo Quijada

Centro de Investigacion y de Estudios Avanzados del IPN, Mexico City, Mexico M.C. Duran-Osuna, H. Castilla-Valdez, E. De La Cruz-Burelo, G. Ramirez-Sanchez, I. Heredia-De La Cruz ${ }^{32}$, R.I. Rabadan-Trejo, R. Lopez-Fernandez, J. Mejia Guisao, R Reyes-Almanza, M. Ramirez-Garcia, A. Sanchez-Hernandez

Universidad Iberoamericana, Mexico City, Mexico

S. Carrillo Moreno, C. Oropeza Barrera, F. Vazquez Valencia

Benemerita Universidad Autonoma de Puebla, Puebla, Mexico

J. Eysermans, I. Pedraza, H.A. Salazar Ibarguen, C. Uribe Estrada

Universidad Autónoma de San Luis Potosí, San Luis Potosí, Mexico

A. Morelos Pineda

University of Auckland, Auckland, New Zealand

D. Krofcheck

University of Canterbury, Christchurch, New Zealand

S. Bheesette, P.H. Butler

National Centre for Physics, Quaid-I-Azam University, Islamabad, Pakistan

A. Ahmad, M. Ahmad, M.I. Asghar, Q. Hassan, H.R. Hoorani, A. Saddique, M.A. Shah, M. Shoaib, M. Waqas

National Centre for Nuclear Research, Swierk, Poland

H. Bialkowska, M. Bluj, B. Boimska, T. Frueboes, M. Górski, M. Kazana, K. Nawrocki, M. Szleper, P. Traczyk, P. Zalewski

Institute of Experimental Physics, Faculty of Physics, University of Warsaw, Warsaw, Poland

K. Bunkowski, A. Byszuk ${ }^{33}$, K. Doroba, A. Kalinowski, M. Konecki, J. Krolikowski, M. Misiura, M. Olszewski, A. Pyskir, M. Walczak 
Laboratório de Instrumentação e Física Experimental de Partículas, Lisboa, Portugal

P. Bargassa, C. Beirão Da Cruz E Silva, A. Di Francesco, P. Faccioli, B. Galinhas, M. Gallinaro, J. Hollar, N. Leonardo, L. Lloret Iglesias, M.V. Nemallapudi, J. Seixas, G. Strong, O. Toldaiev, D. Vadruccio, J. Varela

\section{Joint Institute for Nuclear Research, Dubna, Russia}

V. Alexakhin, A. Golunov, I. Golutvin, N. Gorbounov, I. Gorbunov, A. Kamenev, V. Karjavin, A. Lanev, A. Malakhov, V. Matveev ${ }^{34,35}$, P. Moisenz, V. Palichik, V. Perelygin, M. Savina, S. Shmatov, S. Shulha, N. Skatchkov, V. Smirnov, A. Zarubin

Petersburg Nuclear Physics Institute, Gatchina (St. Petersburg), Russia

V. Golovtsov, Y. Ivanov, V. Kim ${ }^{36}$, E. Kuznetsova ${ }^{37}$, P. Levchenko, V. Murzin, V. Oreshkin, I. Smirnov, D. Sosnov, V. Sulimov, L. Uvarov, S. Vavilov, A. Vorobyev

Institute for Nuclear Research, Moscow, Russia

Yu. Andreev, A. Dermenev, S. Gninenko, N. Golubev, A. Karneyeu, M. Kirsanov, N. Krasnikov, A. Pashenkov, D. Tlisov, A. Toropin

Institute for Theoretical and Experimental Physics, Moscow, Russia

V. Epshteyn, V. Gavrilov, N. Lychkovskaya, V. Popov, I. Pozdnyakov, G. Safronov, A. Spiridonov, A. Stepennov, V. Stolin, M. Toms, E. Vlasov, A. Zhokin

Moscow Institute of Physics and Technology, Moscow, Russia

T. Aushev

National Research Nuclear University 'Moscow Engineering Physics Institute' (MEPhI), Moscow, Russia

M. Chadeeva ${ }^{38}$, P. Parygin, D. Philippov, S. Polikarpov ${ }^{38}$, E. Popova, V. Rusinov

P.N. Lebedev Physical Institute, Moscow, Russia

V. Andreev, M. Azarkin ${ }^{35}$, I. Dremin ${ }^{35}$, M. Kirakosyan ${ }^{35}$, S.V. Rusakov, A. Terkulov

Skobeltsyn Institute of Nuclear Physics, Lomonosov Moscow State University, Moscow, Russia

A. Baskakov, A. Belyaev, E. Boos, M. Dubinin ${ }^{39}$, L. Dudko, A. Ershov, A. Gribushin,

V. Klyukhin, O. Kodolova, I. Lokhtin, I. Miagkov, S. Obraztsov, S. Petrushanko, V. Savrin,

A. Snigirev

Novosibirsk State University (NSU), Novosibirsk, Russia

V. Blinov ${ }^{40}$, T. Dimova ${ }^{40}$, L. Kardapoltsev ${ }^{40}$, D. Shtol ${ }^{40}$, Y. Skovpen ${ }^{40}$

State Research Center of Russian Federation, Institute for High Energy Physics of NRC 'Kurchatov Institute', Protvino, Russia

I. Azhgirey, I. Bayshev, S. Bitioukov, D. Elumakhov, A. Godizov, V. Kachanov, A. Kalinin,

D. Konstantinov, P. Mandrik, V. Petrov, R. Ryutin, S. Slabospitskii, A. Sobol, S. Troshin,

N. Tyurin, A. Uzunian, A. Volkov 


\section{National Research Tomsk Polytechnic University, Tomsk, Russia}

A. Babaev, S. Baidali

University of Belgrade, Faculty of Physics and Vinca Institute of Nuclear Sciences, Belgrade, Serbia

P. Adzic ${ }^{41}$, P. Cirkovic, D. Devetak, M. Dordevic, J. Milosevic

Centro de Investigaciones Energéticas Medioambientales y Tecnológicas (CIEMAT), Madrid, Spain

J. Alcaraz Maestre, A. Álvarez Fernández, I. Bachiller, M. Barrio Luna, J.A. Brochero Cifuentes, M. Cerrada, N. Colino, B. De La Cruz, A. Delgado Peris, C. Fernandez Bedoya, J.P. Fernández Ramos, J. Flix, M.C. Fouz, O. Gonzalez Lopez, S. Goy Lopez, J.M. Hernandez, M.I. Josa, D. Moran, A. Pérez-Calero Yzquierdo, J. Puerta Pelayo, I. Redondo, L. Romero, M.S. Soares, A. Triossi

Universidad Autónoma de Madrid, Madrid, Spain

C. Albajar, J.F. de Trocóniz

Universidad de Oviedo, Oviedo, Spain

J. Cuevas, C. Erice, J. Fernandez Menendez, S. Folgueras, I. Gonzalez Caballero, J.R. González Fernández, E. Palencia Cortezon, V. Rodríguez Bouza, S. Sanchez Cruz, P. Vischia, J.M. Vizan Garcia

Instituto de Física de Cantabria (IFCA), CSIC-Universidad de Cantabria, Santander, Spain

I.J. Cabrillo, A. Calderon, B. Chazin Quero, J. Duarte Campderros, M. Fernandez, P.J. Fernández Manteca, A. García Alonso, J. Garcia-Ferrero, G. Gomez, A. Lopez Virto, J. Marco, C. Martinez Rivero, P. Martinez Ruiz del Arbol, F. Matorras, J. Piedra Gomez, C. Prieels, T. Rodrigo, A. Ruiz-Jimeno, L. Scodellaro, N. Trevisani, I. Vila, R. Vilar Cortabitarte

\section{CERN, European Organization for Nuclear Research, Geneva, Switzerland}

D. Abbaneo, B. Akgun, E. Auffray, P. Baillon, A.H. Ball, D. Barney, J. Bendavid, M. Bianco, A. Bocci, C. Botta, E. Brondolin, T. Camporesi, M. Cepeda, G. Cerminara, E. Chapon, Y. Chen, G. Cucciati, D. d'Enterria, A. Dabrowski, V. Daponte, A. David, A. De Roeck, N. Deelen, M. Dobson, M. Dünser, N. Dupont, A. ElliottPeisert, P. Everaerts, F. Fallavollita ${ }^{42}$, D. Fasanella, G. Franzoni, J. Fulcher, W. Funk, D. Gigi, A. Gilbert, K. Gill, F. Glege, M. Guilbaud, D. Gulhan, J. Hegeman, V. Innocente, A. Jafari, P. Janot, O. Karacheban ${ }^{18}$, J. Kieseler, A. Kornmayer, M. Krammer ${ }^{1}$, C. Lange, P. Lecoq, C. Lourenço, L. Malgeri, M. Mannelli, F. Meijers, J.A. Merlin, S. Mersi, E. Meschi, P. Milenovic ${ }^{43}$, F. Moortgat, M. Mulders, J. Ngadiuba, S. Orfanelli, L. Orsini, F. Pantaleo ${ }^{15}$, L. Pape, E. Perez, M. Peruzzi, A. Petrilli, G. Petrucciani, A. Pfeiffer, M. Pierini, F.M. Pitters, D. Rabady, A. Racz, T. Reis, G. Rolandi ${ }^{44}$, M. Rovere, H. Sakulin, C. Schäfer, C. Schwick, M. Seidel, M. Selvaggi, A. Sharma, P. Silva, P. Sphicas ${ }^{45}$, A. Stakia, J. Steggemann, M. Tosi, D. Treille, A. Tsirou, V. Veckalns ${ }^{46}$, W.D. Zeuner 
Paul Scherrer Institut, Villigen, Switzerland

L. Caminada ${ }^{47}$, K. Deiters, W. Erdmann, R. Horisberger, Q. Ingram, H.C. Kaestli, D. Kotlinski, U. Langenegger, T. Rohe, S.A. Wiederkehr

ETH Zurich - Institute for Particle Physics and Astrophysics (IPA), Zurich, Switzerland

M. Backhaus, L. Bäni, P. Berger, N. Chernyavskaya, G. Dissertori, M. Dittmar, M. Donegà, C. Dorfer, C. Grab, C. Heidegger, D. Hits, J. Hoss, T. Klijnsma, W. Lustermann, R.A. Manzoni, M. Marionneau, M.T. Meinhard, F. Micheli, P. Musella, F. Nessi-Tedaldi, J. Pata, F. Pauss, G. Perrin, L. Perrozzi, S. Pigazzini, M. Quittnat, D. Ruini, D.A. Sanz Becerra, M. Schönenberger, L. Shchutska, V.R. Tavolaro, K. Theofilatos, M.L. Vesterbacka Olsson, R. Wallny, D.H. Zhu

Universität Zürich, Zurich, Switzerland

T.K. Aarrestad, C. Amsler ${ }^{48}$, D. Brzhechko, M.F. Canelli, A. De Cosa, R. Del Burgo, S. Donato, C. Galloni, T. Hreus, B. Kilminster, I. Neutelings, D. Pinna, G. Rauco, P. Robmann, D. Salerno, K. Schweiger, C. Seitz, Y. Takahashi, A. Zucchetta

\section{National Central University, Chung-Li, Taiwan}

Y.H. Chang, K.y. Cheng, T.H. Doan, Sh. Jain, R. Khurana, C.M. Kuo, W. Lin, A. Pozdnyakov, S.S. Yu

\section{National Taiwan University (NTU), Taipei, Taiwan}

P. Chang, Y. Chao, K.F. Chen, P.H. Chen, W.-S. Hou, Arun Kumar, Y.y. Li, Y.F. Liu, R.-S. Lu, E. Paganis, A. Psallidas, A. Steen, J.f. Tsai

Chulalongkorn University, Faculty of Science, Department of Physics, Bangkok, Thailand

B. Asavapibhop, N. Srimanobhas, N. Suwonjandee

Çukurova University, Physics Department, Science and Art Faculty, Adana, Turkey

A. Bat, F. Boran, S. Cerci ${ }^{49}$, S. Damarseckin, Z.S. Demiroglu, F. Dolek, C. Dozen, I. Dumanoglu, S. Girgis, G. Gokbulut, Y. Guler, E. Gurpinar, I. Hos ${ }^{50}$, C. Isik, E.E. Kangal ${ }^{51}$, O. Kara, A. Kayis Topaksu, U. Kiminsu, M. Oglakci, G. Onengut, K. Ozdemir ${ }^{52}$, S. Ozturk ${ }^{53}$, D. Sunar Cerci ${ }^{49}$, B. Tali ${ }^{49}$, U.G. Tok, S. Turkcapar, I.S. Zorbakir, C. Zorbilmez

Middle East Technical University, Physics Department, Ankara, Turkey

B. Isildak ${ }^{54}$, G. Karapinar ${ }^{55}$, M. Yalvac, M. Zeyrek

Bogazici University, Istanbul, Turkey

I.O. Atakisi, E. Gülmez, M. Kaya ${ }^{56}$, O. Kaya ${ }^{57}$, S. Tekten, E.A. Yetkin ${ }^{58}$

Istanbul Technical University, Istanbul, Turkey

M.N. Agaras, S. Atay, A. Cakir, K. Cankocak, Y. Komurcu, S. Sen ${ }^{59}$ 
Institute for Scintillation Materials of National Academy of Science of Ukraine, Kharkov, Ukraine

B. Grynyov

National Scientific Center, Kharkov Institute of Physics and Technology, Kharkov, Ukraine

L. Levchuk

University of Bristol, Bristol, United Kingdom

F. Ball, L. Beck, J.J. Brooke, D. Burns, E. Clement, D. Cussans, O. Davignon, H. Flacher, J. Goldstein, G.P. Heath, H.F. Heath, L. Kreczko, D.M. Newbold ${ }^{60}$, S. Paramesvaran, B. Penning, T. Sakuma, D. Smith, V.J. Smith, J. Taylor, A. Titterton

\section{Rutherford Appleton Laboratory, Didcot, United Kingdom}

K.W. Bell, A. Belyaev ${ }^{61}$, C. Brew, R.M. Brown, D. Cieri, D.J.A. Cockerill, J.A. Coughlan, K. Harder, S. Harper, J. Linacre, E. Olaiya, D. Petyt, C.H. Shepherd-Themistocleous, A. Thea, I.R. Tomalin, T. Williams, W.J. Womersley

\section{Imperial College, London, United Kingdom}

G. Auzinger, R. Bainbridge, P. Bloch, J. Borg, S. Breeze, O. Buchmuller, A. Bundock, S. Casasso, D. Colling, L. Corpe, P. Dauncey, G. Davies, M. Della Negra, R. Di Maria, Y. Haddad, G. Hall, G. Iles, T. James, M. Komm, C. Laner, L. Lyons, A.-M. Magnan, S. Malik, A. Martelli, J. Nash ${ }^{62}$, A. Nikitenko ${ }^{7}$, V. Palladino, M. Pesaresi, A. Richards, A. Rose, E. Scott, C. Seez, A. Shtipliyski, G. Singh, M. Stoye, T. Strebler, S. Summers, A. Tapper, K. Uchida, T. Virdee ${ }^{15}$, N. Wardle, D. Winterbottom, J. Wright, S.C. Zenz

Brunel University, Uxbridge, United Kingdom

J.E. Cole, P.R. Hobson, A. Khan, P. Kyberd, C.K. Mackay, A. Morton, I.D. Reid, L. Teodorescu, S. Zahid

Baylor University, Waco, U.S.A.

K. Call, J. Dittmann, K. Hatakeyama, H. Liu, C. Madrid, B. Mcmaster, N. Pastika, C. Smith

Catholic University of America, Washington DC, U.S.A.

R. Bartek, A. Dominguez

The University of Alabama, Tuscaloosa, U.S.A.

A. Buccilli, S.I. Cooper, C. Henderson, P. Rumerio, C. West

Boston University, Boston, U.S.A.

D. Arcaro, T. Bose, D. Gastler, D. Rankin, C. Richardson, J. Rohlf, L. Sulak, D. Zou

Brown University, Providence, U.S.A.

G. Benelli, X. Coubez, D. Cutts, M. Hadley, J. Hakala, U. Heintz, J.M. Hogan ${ }^{63}$, K.H.M. Kwok, E. Laird, G. Landsberg, J. Lee, Z. Mao, M. Narain, S. Piperov, S. Sagir ${ }^{64}$, R. Syarif, E. Usai, D. Yu 
University of California, Davis, Davis, U.S.A.

R. Band, C. Brainerd, R. Breedon, D. Burns, M. Calderon De La Barca Sanchez,

M. Chertok, J. Conway, R. Conway, P.T. Cox, R. Erbacher, C. Flores, G. Funk, W. Ko,

O. Kukral, R. Lander, C. Mclean, M. Mulhearn, D. Pellett, J. Pilot, S. Shalhout, M. Shi,

D. Stolp, D. Taylor, K. Tos, M. Tripathi, Z. Wang, F. Zhang

University of California, Los Angeles, U.S.A.

M. Bachtis, C. Bravo, R. Cousins, A. Dasgupta, A. Florent, J. Hauser, M. Ignatenko, N. Mccoll, S. Regnard, D. Saltzberg, C. Schnaible, V. Valuev

University of California, Riverside, Riverside, U.S.A.

E. Bouvier, K. Burt, R. Clare, J.W. Gary, S.M.A. Ghiasi Shirazi, G. Hanson, G. Karapostoli, E. Kennedy, F. Lacroix, O.R. Long, M. Olmedo Negrete, M.I. Paneva, W. Si, L. Wang, H. Wei, S. Wimpenny, B.R. Yates

University of California, San Diego, La Jolla, U.S.A.

J.G. Branson, S. Cittolin, M. Derdzinski, R. Gerosa, D. Gilbert, B. Hashemi, A. Holzner, D. Klein, G. Kole, V. Krutelyov, J. Letts, M. Masciovecchio, D. Olivito, S. Padhi, M. Pieri, M. Sani, V. Sharma, S. Simon, M. Tadel, A. Vartak, S. Wasserbaech ${ }^{65}$, J. Wood, F. Würthwein, A. Yagil, G. Zevi Della Porta

University of California, Santa Barbara - Department of Physics, Santa Barbara, U.S.A.

N. Amin, R. Bhandari, J. Bradmiller-Feld, C. Campagnari, M. Citron, A. Dishaw, V. Dutta, M. Franco Sevilla, L. Gouskos, R. Heller, J. Incandela, A. Ovcharova, H. Qu, J. Richman, D. Stuart, I. Suarez, S. Wang, J. Yoo

California Institute of Technology, Pasadena, U.S.A.

D. Anderson, A. Bornheim, J.M. Lawhorn, H.B. Newman, T.Q. Nguyen, M. Spiropulu, J.R. Vlimant, R. Wilkinson, S. Xie, Z. Zhang, R.Y. Zhu

Carnegie Mellon University, Pittsburgh, U.S.A.

M.B. Andrews, T. Ferguson, T. Mudholkar, M. Paulini, M. Sun, I. Vorobiev, M. Weinberg

University of Colorado Boulder, Boulder, U.S.A.

J.P. Cumalat, W.T. Ford, F. Jensen, A. Johnson, M. Krohn, S. Leontsinis, E. MacDonald, T. Mulholland, K. Stenson, K.A. Ulmer, S.R. Wagner

Cornell University, Ithaca, U.S.A.

J. Alexander, J. Chaves, Y. Cheng, J. Chu, A. Datta, K. Mcdermott, N. Mirman, J.R. Patterson, D. Quach, A. Rinkevicius, A. Ryd, L. Skinnari, L. Soffi, S.M. Tan, Z. Tao, J. Thom, J. Tucker, P. Wittich, M. Zientek

\section{Fermi National Accelerator Laboratory, Batavia, U.S.A.}

S. Abdullin, M. Albrow, M. Alyari, G. Apollinari, A. Apresyan, A. Apyan, S. Banerjee, L.A.T. Bauerdick, A. Beretvas, J. Berryhill, P.C. Bhat, G. Bolla ${ }^{\dagger}$, K. Burkett, J.N. Butler, A. Canepa, G.B. Cerati, H.W.K. Cheung, F. Chlebana, M. Cremonesi, J. Duarte, V.D. Elvira, J. Freeman, Z. Gecse, E. Gottschalk, L. Gray, D. Green, S. Grünendahl, 
O. Gutsche, J. Hanlon, R.M. Harris, S. Hasegawa, J. Hirschauer, Z. Hu, B. Jayatilaka, S. Jindariani, M. Johnson, U. Joshi, B. Klima, M.J. Kortelainen, B. Kreis, S. Lammel, D. Lincoln, R. Lipton, M. Liu, T. Liu, J. Lykken, K. Maeshima, J.M. Marraffino, D. Mason, P. McBride, P. Merkel, S. Mrenna, S. Nahn, V. O'Dell, K. Pedro, O. Prokofyev, G. Rakness, L. Ristori, A. Savoy-Navarro ${ }^{66}$, B. Schneider, E. Sexton-Kennedy, A. Soha, W.J. Spalding, L. Spiegel, S. Stoynev, J. Strait, N. Strobbe, L. Taylor, S. Tkaczyk, N.V. Tran, L. Uplegger, E.W. Vaandering, C. Vernieri, M. Verzocchi, R. Vidal, M. Wang, H.A. Weber, A. Whitbeck

University of Florida, Gainesville, U.S.A.

D. Acosta, P. Avery, P. Bortignon, D. Bourilkov, A. Brinkerhoff, L. Cadamuro, A. Carnes, M. Carver, D. Curry, R.D. Field, S.V. Gleyzer, B.M. Joshi, J. Konigsberg, A. Korytov, P. Ma, K. Matchev, H. Mei, G. Mitselmakher, K. Shi, D. Sperka, J. Wang, S. Wang

Florida International University, Miami, U.S.A.

Y.R. Joshi, S. Linn

Florida State University, Tallahassee, U.S.A.

A. Ackert, T. Adams, A. Askew, S. Hagopian, V. Hagopian, K.F. Johnson, T. Kolberg, G. Martinez, T. Perry, H. Prosper, A. Saha, V. Sharma, R. Yohay

Florida Institute of Technology, Melbourne, U.S.A.

M.M. Baarmand, V. Bhopatkar, S. Colafranceschi, M. Hohlmann, D. Noonan, M. Rahmani, T. Roy, F. Yumiceva

University of Illinois at Chicago (UIC), Chicago, U.S.A.

M.R. Adams, L. Apanasevich, D. Berry, R.R. Betts, R. Cavanaugh, X. Chen, S. Dittmer, O. Evdokimov, C.E. Gerber, D.A. Hangal, D.J. Hofman, K. Jung, J. Kamin, C. Mills, I.D. Sandoval Gonzalez, M.B. Tonjes, N. Varelas, H. Wang, X. Wang, Z. Wu, J. Zhang

The University of Iowa, Iowa City, U.S.A.

M. Alhusseini, B. Bilki ${ }^{67}$, W. Clarida, K. Dilsiz ${ }^{68}$, S. Durgut, R.P. Gandrajula, M. Haytmyradov, V. Khristenko, J.-P. Merlo, A. Mestvirishvili, A. Moeller, J. Nachtman, H. Ogul ${ }^{69}$, Y. Onel, F. Ozok ${ }^{70}$, A. Penzo, C. Snyder, E. Tiras, J. Wetzel

Johns Hopkins University, Baltimore, U.S.A.

B. Blumenfeld, A. Cocoros, N. Eminizer, D. Fehling, L. Feng, A.V. Gritsan, W.T. Hung, P. Maksimovic, J. Roskes, U. Sarica, M. Swartz, M. Xiao, C. You

The University of Kansas, Lawrence, U.S.A.

A. Al-bataineh, P. Baringer, A. Bean, S. Boren, J. Bowen, A. Bylinkin, J. Castle, S. Khalil,

A. Kropivnitskaya, D. Majumder, W. Mcbrayer, M. Murray, C. Rogan, S. Sanders,

E. Schmitz, J.D. Tapia Takaki, Q. Wang

Kansas State University, Manhattan, U.S.A.

S. Duric, A. Ivanov, K. Kaadze, D. Kim, Y. Maravin, D.R. Mendis, T. Mitchell, A. Modak, A. Mohammadi, L.K. Saini, N. Skhirtladze

Lawrence Livermore National Laboratory, Livermore, U.S.A.

F. Rebassoo, D. Wright 
University of Maryland, College Park, U.S.A.

A. Baden, O. Baron, A. Belloni, S.C. Eno, Y. Feng, C. Ferraioli, N.J. Hadley, S. Jabeen, G.Y. Jeng, R.G. Kellogg, J. Kunkle, A.C. Mignerey, F. Ricci-Tam, Y.H. Shin, A. Skuja, S.C. Tonwar, K. Wong

\section{Massachusetts Institute of Technology, Cambridge, U.S.A.}

D. Abercrombie, B. Allen, V. Azzolini, A. Baty, G. Bauer, R. Bi, S. Brandt, W. Busza, I.A. Cali, M. D'Alfonso, Z. Demiragli, G. Gomez Ceballos, M. Goncharov, P. Harris, D. Hsu, M. Hu, Y. Iiyama, G.M. Innocenti, M. Klute, D. Kovalskyi, Y.-J. Lee, P.D. Luckey, B. Maier, A.C. Marini, C. Mcginn, C. Mironov, S. Narayanan, X. Niu, C. Paus, C. Roland, G. Roland, G.S.F. Stephans, K. Sumorok, K. Tatar, D. Velicanu, J. Wang, T.W. Wang, B. Wyslouch, S. Zhaozhong

University of Minnesota, Minneapolis, U.S.A.

A.C. Benvenuti, R.M. Chatterjee, A. Evans, P. Hansen, S. Kalafut, Y. Kubota, Z. Lesko, J. Mans, S. Nourbakhsh, N. Ruckstuhl, R. Rusack, J. Turkewitz, M.A. Wadud

University of Mississippi, Oxford, U.S.A.

J.G. Acosta, S. Oliveros

University of Nebraska-Lincoln, Lincoln, U.S.A.

E. Avdeeva, K. Bloom, D.R. Claes, C. Fangmeier, F. Golf, R. Gonzalez Suarez, R. Kamalieddin, I. Kravchenko, J. Monroy, J.E. Siado, G.R. Snow, B. Stieger

State University of New York at Buffalo, Buffalo, U.S.A.

A. Godshalk, C. Harrington, I. Iashvili, A. Kharchilava, D. Nguyen, A. Parker, S. Rappoccio, B. Roozbahani

Northeastern University, Boston, U.S.A.

E. Barberis, C. Freer, A. Hortiangtham, D.M. Morse, T. Orimoto, R. Teixeira De Lima, T. Wamorkar, B. Wang, A. Wisecarver, D. Wood

Northwestern University, Evanston, U.S.A.

S. Bhattacharya, O. Charaf, K.A. Hahn, N. Mucia, N. Odell, M.H. Schmitt, K. Sung, M. Trovato, M. Velasco

University of Notre Dame, Notre Dame, U.S.A.

R. Bucci, N. Dev, M. Hildreth, K. Hurtado Anampa, C. Jessop, D.J. Karmgard, N. Kellams, K. Lannon, W. Li, N. Loukas, N. Marinelli, F. Meng, C. Mueller, Y. Musienko ${ }^{34}$, M. Planer, A. Reinsvold, R. Ruchti, P. Siddireddy, G. Smith, S. Taroni, M. Wayne, A. Wightman, M. Wolf, A. Woodard

The Ohio State University, Columbus, U.S.A.

J. Alimena, L. Antonelli, B. Bylsma, L.S. Durkin, S. Flowers, B. Francis, A. Hart, C. Hill, W. Ji, T.Y. Ling, W. Luo, B.L. Winer, H.W. Wulsin 
Princeton University, Princeton, U.S.A.

S. Cooperstein, P. Elmer, J. Hardenbrook, P. Hebda, S. Higginbotham, A. Kalogeropoulos,

D. Lange, M.T. Lucchini, J. Luo, D. Marlow, K. Mei, I. Ojalvo, J. Olsen, C. Palmer, P. Piroué, J. Salfeld-Nebgen, D. Stickland, C. Tully

University of Puerto Rico, Mayaguez, U.S.A.

S. Malik, S. Norberg

Purdue University, West Lafayette, U.S.A.

A. Barker, V.E. Barnes, S. Das, L. Gutay, M. Jones, A.W. Jung, A. Khatiwada, B. Mahakud, D.H. Miller, N. Neumeister, C.C. Peng, H. Qiu, J.F. Schulte, J. Sun, F. Wang, R. Xiao, W. Xie

Purdue University Northwest, Hammond, U.S.A.

T. Cheng, J. Dolen, N. Parashar

Rice University, Houston, U.S.A.

Z. Chen, K.M. Ecklund, S. Freed, F.J.M. Geurts, M. Kilpatrick, W. Li, B. Michlin, B.P. Padley, J. Roberts, J. Rorie, W. Shi, Z. Tu, J. Zabel, A. Zhang

University of Rochester, Rochester, U.S.A.

A. Bodek, P. de Barbaro, R. Demina, Y.t. Duh, J.L. Dulemba, C. Fallon, T. Ferbel, M. Galanti, A. Garcia-Bellido, J. Han, O. Hindrichs, A. Khukhunaishvili, K.H. Lo, P. Tan, R. Taus, M. Verzetti

Rutgers, The State University of New Jersey, Piscataway, U.S.A.

A. Agapitos, J.P. Chou, Y. Gershtein, T.A. Gómez Espinosa, E. Halkiadakis, M. Heindl, E. Hughes, S. Kaplan, R. Kunnawalkam Elayavalli, S. Kyriacou, A. Lath, R. Montalvo, K. Nash, M. Osherson, H. Saka, S. Salur, S. Schnetzer, D. Sheffield, S. Somalwar, R. Stone, S. Thomas, P. Thomassen, M. Walker

University of Tennessee, Knoxville, U.S.A.

A.G. Delannoy, J. Heideman, G. Riley, K. Rose, S. Spanier, K. Thapa

Texas A\&M University, College Station, U.S.A.

O. Bouhali ${ }^{71}$, A. Celik, M. Dalchenko, M. De Mattia, A. Delgado, S. Dildick, R. Eusebi, J. Gilmore, T. Huang, T. Kamon ${ }^{72}$, S. Luo, R. Mueller, Y. Pakhotin, R. Patel, A. Perloff, L. Perniè, D. Rathjens, A. Safonov, A. Tatarinov

\section{Texas Tech University, Lubbock, U.S.A.}

N. Akchurin, J. Damgov, F. De Guio, P.R. Dudero, S. Kunori, K. Lamichhane, S.W. Lee, T. Mengke, S. Muthumuni, T. Peltola, S. Undleeb, I. Volobouev, Z. Wang

Vanderbilt University, Nashville, U.S.A.

S. Greene, A. Gurrola, R. Janjam, W. Johns, C. Maguire, A. Melo, H. Ni, K. Padeken, J.D. Ruiz Alvarez, P. Sheldon, S. Tuo, J. Velkovska, M. Verweij, Q. Xu 


\section{University of Virginia, Charlottesville, U.S.A.}

M.W. Arenton, P. Barria, B. Cox, R. Hirosky, M. Joyce, A. Ledovskoy, H. Li, C. Neu, T. Sinthuprasith, Y. Wang, E. Wolfe, F. Xia

\section{Wayne State University, Detroit, U.S.A.}

R. Harr, P.E. Karchin, N. Poudyal, J. Sturdy, P. Thapa, S. Zaleski

University of Wisconsin - Madison, Madison, WI, U.S.A.

M. Brodski, J. Buchanan, C. Caillol, D. Carlsmith, S. Dasu, L. Dodd, B. Gomber, M. Grothe, M. Herndon, A. Hervé, U. Hussain, P. Klabbers, A. Lanaro, A. Levine, K. Long, R. Loveless, T. Ruggles, A. Savin, N. Smith, W.H. Smith, N. Woods

\section{t: Deceased}

1: Also at Vienna University of Technology, Vienna, Austria

2: Also at IRFU, CEA, Université Paris-Saclay, Gif-sur-Yvette, France

3: Also at Universidade Estadual de Campinas, Campinas, Brazil

4: Also at Federal University of Rio Grande do Sul, Porto Alegre, Brazil

5: Also at Université Libre de Bruxelles, Bruxelles, Belgium

6: Also at University of Chinese Academy of Sciences, Beijing, China

7: Also at Institute for Theoretical and Experimental Physics, Moscow, Russia

8: Also at Joint Institute for Nuclear Research, Dubna, Russia

9: Also at Cairo University, Cairo, Egypt

10: Also at Helwan University, Cairo, Egypt

11: Now at Zewail City of Science and Technology, Zewail, Egypt

12: Also at Department of Physics, King Abdulaziz University, Jeddah, Saudi Arabia

13: Also at Université de Haute Alsace, Mulhouse, France

14: Also at Skobeltsyn Institute of Nuclear Physics, Lomonosov Moscow State University, Moscow, Russia

15: Also at CERN, European Organization for Nuclear Research, Geneva, Switzerland

16: Also at RWTH Aachen University, III. Physikalisches Institut A, Aachen, Germany

17: Also at University of Hamburg, Hamburg, Germany

18: Also at Brandenburg University of Technology, Cottbus, Germany

19: Also at MTA-ELTE Lendület CMS Particle and Nuclear Physics Group, Eötvös Loránd University, Budapest, Hungary

20: Also at Institute of Nuclear Research ATOMKI, Debrecen, Hungary

21: Also at Institute of Physics, University of Debrecen, Debrecen, Hungary

22: Also at Indian Institute of Technology Bhubaneswar, Bhubaneswar, India

23: Also at Institute of Physics, Bhubaneswar, India

24: Also at Shoolini University, Solan, India

25: Also at University of Visva-Bharati, Santiniketan, India

26: Also at Isfahan University of Technology, Isfahan, Iran

27: Also at Plasma Physics Research Center, Science and Research Branch, Islamic Azad University, Tehran, Iran

28: Also at Università degli Studi di Siena, Siena, Italy

29: Also at Kyunghee University, Seoul, Korea

30: Also at International Islamic University of Malaysia, Kuala Lumpur, Malaysia

31: Also at Malaysian Nuclear Agency, MOSTI, Kajang, Malaysia

32: Also at Consejo Nacional de Ciencia y Tecnología, Mexico city, Mexico 
33: Also at Warsaw University of Technology, Institute of Electronic Systems, Warsaw, Poland

34: Also at Institute for Nuclear Research, Moscow, Russia

35: Now at National Research Nuclear University 'Moscow Engineering Physics Institute' (MEPhI), Moscow, Russia

36: Also at St. Petersburg State Polytechnical University, St. Petersburg, Russia

37: Also at University of Florida, Gainesville, U.S.A.

38: Also at P.N. Lebedev Physical Institute, Moscow, Russia

39: Also at California Institute of Technology, Pasadena, U.S.A.

40: Also at Budker Institute of Nuclear Physics, Novosibirsk, Russia

41: Also at Faculty of Physics, University of Belgrade, Belgrade, Serbia

42: Also at INFN Sezione di Pavia ${ }^{a}$, Università di Pavia ${ }^{b}$, Pavia, Italy

43: Also at University of Belgrade, Faculty of Physics and Vinca Institute of Nuclear Sciences, Belgrade, Serbia

44: Also at Scuola Normale e Sezione dell'INFN, Pisa, Italy

45: Also at National and Kapodistrian University of Athens, Athens, Greece

46: Also at Riga Technical University, Riga, Latvia

47: Also at Universität Zürich, Zurich, Switzerland

48: Also at Stefan Meyer Institute for Subatomic Physics (SMI), Vienna, Austria

49: Also at Adiyaman University, Adiyaman, Turkey

50: Also at Istanbul Aydin University, Istanbul, Turkey

51: Also at Mersin University, Mersin, Turkey

52: Also at Piri Reis University, Istanbul, Turkey

53: Also at Gaziosmanpasa University, Tokat, Turkey

54: Also at Ozyegin University, Istanbul, Turkey

55: Also at Izmir Institute of Technology, Izmir, Turkey

56: Also at Marmara University, Istanbul, Turkey

57: Also at Kafkas University, Kars, Turkey

58: Also at Istanbul Bilgi University, Istanbul, Turkey

59: Also at Hacettepe University, Ankara, Turkey

60: Also at Rutherford Appleton Laboratory, Didcot, United Kingdom

61: Also at School of Physics and Astronomy, University of Southampton, Southampton, United Kingdom

62: Also at Monash University, Faculty of Science, Clayton, Australia

63: Also at Bethel University, St. Paul, U.S.A.

64: Also at Karamanoğlu Mehmetbey University, Karaman, Turkey

65: Also at Utah Valley University, Orem, U.S.A.

66: Also at Purdue University, West Lafayette, U.S.A.

67: Also at Beykent University, Istanbul, Turkey

68: Also at Bingol University, Bingol, Turkey

69: Also at Sinop University, Sinop, Turkey

70: Also at Mimar Sinan University, Istanbul, Istanbul, Turkey

71: Also at Texas A\&M University at Qatar, Doha, Qatar

72: Also at Kyungpook National University, Daegu, Korea 